\title{
Synthesis of 1,2-Dioxolanes by Annulation Reactions of Peroxycarbenium Ions with Alkenes
}

\author{
Armando Ramirez and K. A. Woerpel* \\ Department of Chemistry, University of California \\ Irvine, CA 92697-2025
}

\section{Supporting Information}

\section{Contents:}
I. Preparation of Peroxyketal and Silyl Peroxyketals
$S-1$
II. Preparation of Alkenes
III. Annulation Products Between Peroxycarbenium ions and Alkenes
IV. Oxidation of Silane Moiety
V. Oxidation of Hydroxyl Groups
S-6
VI. Stereochemical Proofs of Annulation Products
$S-11$
VII. X-Ray Crystallographic Data
$S-13$
VIII. Bibliography
$S-14$
$S-15$
IX. Analytical Data
$S-23$
$S-24$

General. Melting points were obtained using a Büchi 510 melting point apparatus and are reported uncorrected. ${ }^{1} \mathrm{H}$ NMR and ${ }^{13} \mathrm{C}$ NMR spectra were recorded at ambient temperature at $500 \mathrm{MHz}$ and $400 \mathrm{MHz}$ and $125 \mathrm{MHz}$ and $100 \mathrm{MHz}$, respectively, using Bruker DRX 500 or DRX 400 spectrometers. The data are reported as follows: chemical shift in ppm from internal tetramethylsilane on the $\delta$ scale, multiplicity (br $=$ broad, $\mathrm{s}=$ singlet, $\mathrm{d}=$ doublet, $\mathrm{t}=$ triplet, $\mathrm{q}=$ quartet, $\mathrm{m}=$ multiplet $)$, coupling constants $(\mathrm{Hz})$, and integration. Infrared (IR) spectra were obtained using a MIDAC Prospect FT-IR spectrometer. High-resolution mass spectra were acquired on a VG Analytical 7070E or Fisons Autospec spectrometer, and were obtained by peak matching. Microanalyses were performed by Atlantic Microlab, Atlanta, GA. Liquid chromatography was performed using forced flow (flash chromatography) of the indicated solvent system on EM Reagents silica gel $\left(\mathrm{SiO}_{2}\right) 60$ (230-400 mesh). As needed, reactions were performed under an atmosphere of nitrogen and flash chromatography was performed using Aldrich Davisil ${ }^{\circledR}$ silica gel $\left(\mathrm{SiO}_{2}\right) 60$ (200-425 mesh) grade 633. Unless otherwise noted, all reagents were commercially obtained and, where appropriate, purified prior to use. THF, $\mathrm{Et}_{2} \mathrm{O}$, and $\mathrm{CH}_{2} \mathrm{Cl}_{2}$ were dried by filtration through alumina. ${ }^{1}$

Caution: When handling organic peroxides minimize exposure to light, heat and metal salts. ${ }^{2-3}$ Furthermore, perform reactions in a small scale, and employ a safety shield.

\section{Synthesis of the Peroxyketal and Silyl Peroxyketals}

General Procedure for the Silyl Protection of Peroxyketals. Unless otherwise noted a solution of peroxyketal (1.0 equiv) and DMF $(0.4-0.98 \mathrm{M})$ was treated with chlorosilane or trifluoromethanesulfonatesilane $(2.2-3.0$ equiv $)$ and triethylamine $(2.0-6.0$ equiv $)$. The reaction mixture was stirred for $10 \mathrm{~min}-24 \mathrm{~h}$ at $\left(-40{ }^{\circ} \mathrm{C}-25^{\circ} \mathrm{C}\right)$ then added to ice-cold $\mathrm{H}_{2} \mathrm{O}$. The reaction mixture was stirred for $5 \mathrm{~min}$ and $100 \mathrm{~mL}$ of brine was added. The mixture was extracted with $4 \times 100 \mathrm{~mL}$ of pentane. The organic layers were combined, dried over $\mathrm{Na}_{2} \mathrm{SO}_{4}$, filtered, and the solvent was removed in vacuo. Purification by flash chromatography was performed using Davisil ${ }^{\circledR}$ silica gel. 


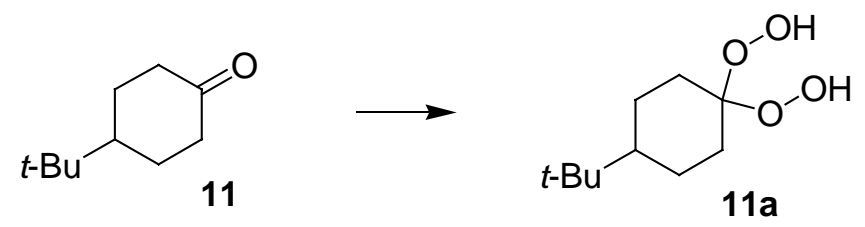

Peroxyketal (11a). ${ }^{4}$ A solution of 4-tert-butylcyclohexanone 11 (5.00 g, 32.4 mmol), molecular sieves $(5.00 \mathrm{~g}, 3 \AA$ ) $)$ and $150 \mathrm{~mL}$ of $\mathrm{CH}_{2} \mathrm{Cl}_{2}$, was treated with $185 \mathrm{~mL}$ of formic acid and $\mathrm{H}_{2} \mathrm{O}_{2}(45 \mathrm{~mL}, 1000 \mathrm{mmol}$, $50 \% \mathrm{H}_{2} \mathrm{O}$ ). The reaction mixture was stirred vigorously for $3 \mathrm{~min}$ at ambient temperature then added to $50 \mathrm{~mL}$

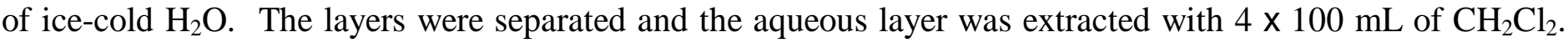
The organic layers were combined and solvent was removed in vacuo to afford peroxyketal 11a as a white powder (6.6 g, 99\%): $\mathrm{mp} 83.0-84.0{ }^{\circ} \mathrm{C} ;{ }^{1} \mathrm{H}$ NMR $\left(500 \mathrm{MHz}, \mathrm{CDCl}_{3}\right) \delta 8.27(\mathrm{~s}, 2 \mathrm{H}), 2.30(\mathrm{~d}, J=13.4,2 \mathrm{H}), 1.70$ $(\mathrm{d}, J=12.7,2 \mathrm{H}), 1.43(\mathrm{t}, J=13.5,2 \mathrm{H}) 1.24(\mathrm{~m}, 2 \mathrm{H}), 1.06(\mathrm{~s}, 1 \mathrm{H}), 0.87(\mathrm{~s}, 9 \mathrm{H}) ;{ }^{13} \mathrm{C} \mathrm{NMR}\left(125 \mathrm{MHz}, \mathrm{CDCl}_{3}\right) \delta$ 110.9, 47.7, 32.5, 29.9, 27.8, 23.6; IR $(\mathrm{KBr}) 3422,2956 \mathrm{~cm}^{-1}$; HRMS $\left(\mathrm{CI} / \mathrm{NH}_{3}\right) \mathrm{m} / z$ calcd for $\mathrm{C}_{10} \mathrm{H}_{19} \mathrm{O}_{2}(\mathrm{M})$ 171.1380, found 171.1381. Anal. Calcd for $\mathrm{C}_{10} \mathrm{H}_{20} \mathrm{O}_{4}: \mathrm{C}, 58.80 ; \mathrm{H}, 9.87$. Found: C, 59.03; H, 9.96.
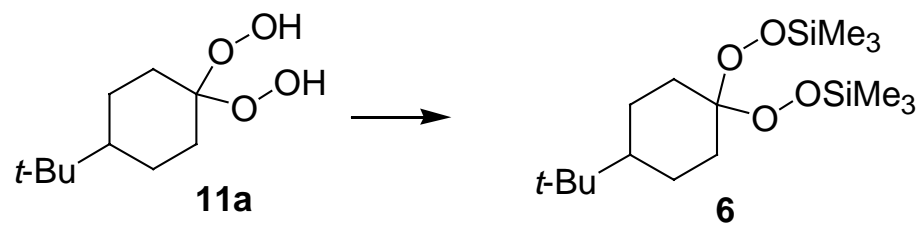

Trimethylsilylperoxyketal (6). The standard silyl protection procedure was followed with $\mathbf{1 1 a}(1.56 \mathrm{~g}$, $7.64 \mathrm{mmol})$ in $88 \mathrm{~mL}$ of DMF with trimethylchlorosilane $(2.15 \mathrm{~mL}, 16.8 \mathrm{mmol})$ and triethylamine $(2.16 \mathrm{~mL}$, $15.3 \mathrm{mmol}){ }^{5}$ The solvent was removed in vacuo to afford 6 as a white solid $(2.60 \mathrm{~g}, 98 \%): \mathrm{mp} 54.0-55.0^{\circ} \mathrm{C}$; ${ }^{1} \mathrm{H}$ NMR $\left(500 \mathrm{MHz}, \mathrm{CDCl}_{3}\right) \delta 2.31(\mathrm{~m}, 2 \mathrm{H}), 1.62(\mathrm{~m}, 2 \mathrm{H}), 1.35(\mathrm{~m}, 2 \mathrm{H}), 1.22(\mathrm{~m}, 2 \mathrm{H}), 1.02(\mathrm{~m}, 1 \mathrm{H}), 0.86(\mathrm{~s}$, 9H), 0.23 (s, 9H), 0.19 (s, 9H); ${ }^{13} \mathrm{C}$ NMR $\left(125 \mathrm{MHz}, \mathrm{CDCl}_{3}\right) \delta 109.6,47.7,32.5,30.7,27.8,23.7,-0.8,-1.0 ; \mathrm{IR}$ (thin film) 2962, 2868, $1250 \mathrm{~cm}^{-1}$; HRMS (ESI/Na) $m / z$ calcd for $\mathrm{C}_{16} \mathrm{H}_{36} \mathrm{O}_{4} \mathrm{Si}_{2} \mathrm{Na}(\mathrm{M}+\mathrm{Na}) 371.2050$, found 371.2058. Anal. Calcd for $\mathrm{C}_{16} \mathrm{H}_{36} \mathrm{O}_{4} \mathrm{Si}_{2}$ : C, 55.12; H, 10.41. Found: C, 56.62; H, 10.44 .
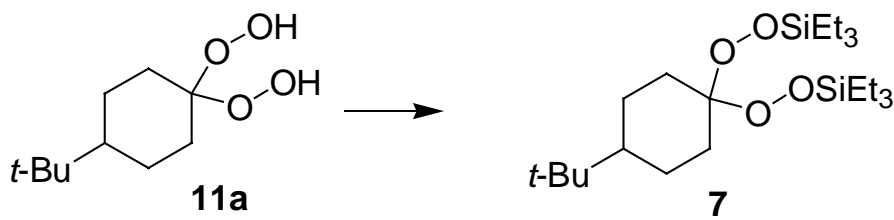

Trimethylsilylperoxyketal (7). The standard silyl protection procedure was followed with $11 \mathbf{a}(0.50 \mathrm{~g}$, $2.5 \mathrm{mmol})$ in $33 \mathrm{~mL}$ of DMF with triethylchlorosilane $(0.91 \mathrm{~mL}, 5.4 \mathrm{mmol})$ and triethylamine $(0.76 \mathrm{~mL}, 5.4$ mmol). Purification by flash chromatography (hexanes) afforded 7 as a clear liquid $(0.77 \mathrm{~g}, 73 \%)$ : ${ }^{1} \mathrm{H}$ NMR $\left(500 \mathrm{MHz}, \mathrm{C}_{6} \mathrm{D}_{6}\right) \delta 2.61(\mathrm{~m}, 2 \mathrm{H}), 1.56(\mathrm{~m}, 2 \mathrm{H}), 1.39(\mathrm{~m}, 4 \mathrm{H}), 1.15(\mathrm{t}, J=7.9,9 \mathrm{H}), 1.11(\mathrm{t}, J=7.9,9 \mathrm{H}), 0.87$ $(\mathrm{m}, 1 \mathrm{H}), 0.86(\mathrm{q}, J=8.0,6 \mathrm{H}), 0.83(\mathrm{~s}, 9 \mathrm{H}), 0.80(\mathrm{q}, J=8.1,6 \mathrm{H}) ;{ }^{13} \mathrm{C} \mathrm{NMR}\left(125 \mathrm{MHz}, \mathrm{C}_{6} \mathrm{D}_{6}\right) \delta 109.9,48.1$, 32.7, 31.4, 28.1, 24.4, 7.5, 4.80, 4.76; IR (thin film) 2951, 2874, 1448, 1238, $1012 \mathrm{~cm}^{-1} ; \mathrm{HRMS}\left(\mathrm{CI} / \mathrm{NH}_{3}\right) \mathrm{m} / z$ calcd for $\mathrm{C}_{20} \mathrm{H}_{43} \mathrm{O}_{4} \mathrm{Si}_{2}(\mathrm{M}-\mathrm{Et})$ 403.2700, found 403.2702. Anal. Calcd for $\mathrm{C}_{22} \mathrm{H}_{48} \mathrm{O}_{4} \mathrm{Si}_{2}$ : C, 61.05; $\mathrm{H}, 11.18$. Found: C, 61.23; H, 11.29. 


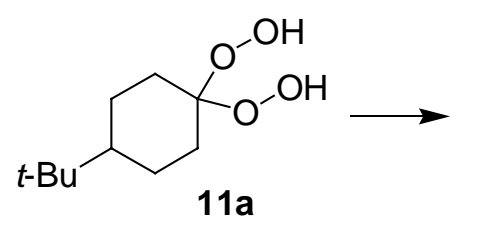<smiles>CCCCOOC1(OOC)CCC(C(C)(C)C)CC1</smiles>

tert-Butyldimethylsilylperoxyketal (8). The standard silyl protection procedure was followed with 11a $(1.50 \mathrm{~g}, 7.35 \mathrm{mmol})$ in $75 \mathrm{~mL}$ of DMF was cooled to $-10{ }^{\circ} \mathrm{C}$. The mixture was treated with tertbutyldimethylsilyl trifluoromethanesulfonate $(3.40 \mathrm{~mL}, 14.7 \mathrm{mmol})$ and triethylamine $(2.10 \mathrm{~mL}, 14.7 \mathrm{mmol})$. Purification by flash chromatography (100:0 - 90:10 hexanes/EtOAc ) afforded 8 as a clear viscous oil (1.7 g, 53\%): ${ }^{1} \mathrm{H}$ NMR $\left(500 \mathrm{MHz}, \mathrm{CDCl}_{3}\right) \delta 2.30(\mathrm{~m}, 2 \mathrm{H}), 1.63(\mathrm{~m}, 2 \mathrm{H}), 1.35-1.22(\mathrm{~m}, 4 \mathrm{H}), 1.02(\mathrm{~m}, 1 \mathrm{H}), 0.96(\mathrm{~s}, 9 \mathrm{H})$, $0.93(\mathrm{~s}, 9 \mathrm{H}), 0.86(\mathrm{~s}, 9 \mathrm{H}), 0.18(\mathrm{~s}, 6 \mathrm{H}), 0.15(\mathrm{~s}, 6 \mathrm{H}) ;{ }^{13} \mathrm{C} \mathrm{NMR}\left(125 \mathrm{MHz}, \mathrm{CDCl}_{3}\right) \delta 109.4,47.7,32.5,30.6$, 27.8, 26.43, 26.36, 23.7, 18.6, 18.4, -5.36, -5.39; IR (thin film) 2954, 2858, 1445, 1250, $1061 \mathrm{~cm}^{-1}$; HRMS $(\mathrm{ESI} / \mathrm{Na}) \mathrm{m} / \mathrm{z}$ calcd for $\mathrm{C}_{22} \mathrm{H}_{48} \mathrm{O}_{4} \mathrm{Si}_{2} \mathrm{Na}(\mathrm{M}+\mathrm{Na})$ 455.2989, found 455.2977. Anal. Calcd for $\mathrm{C}_{22} \mathrm{H}_{48} \mathrm{O}_{4} \mathrm{Si}_{2}: \mathrm{C}_{\text {, }}$ 61.05; H, 11.18. Found: C, 61.32; H, 11.27.
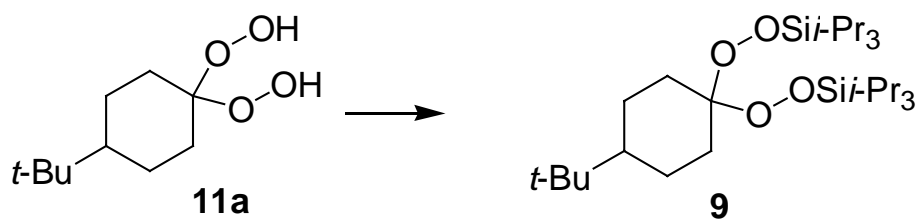

Triiso-propylsilylperoxyketal (9). The standard silyl protection procedure was followed with 11a $(1.00 \mathrm{~g}, 4.9 \mathrm{mmol})$ in $50 \mathrm{~mL}$ of DMF was cooled to $-40{ }^{\circ} \mathrm{C}$. The mixture was treated with triiso-propylsilyl trifluoromethanesulfonate $(3.95 \mathrm{~mL}, 14.7 \mathrm{mmol})$ and triethylamine $(4.20 \mathrm{~mL}, 29.4 \mathrm{mmol})$. Purification by flash chromatography (hexanes) afforded 9 as a clear liquid $(1.10 \mathrm{~g}, 43 \%):{ }^{1} \mathrm{H} \mathrm{NMR}\left(500 \mathrm{MHz}, \mathrm{CDCl}_{3}\right) \delta 2.35(\mathrm{~m}$, $2 \mathrm{H}), 1.64(\mathrm{~m}, 2 \mathrm{H}), 1.35(\mathrm{~m}, 2 \mathrm{H}), 1.28-1.17(\mathrm{~m}, 9 \mathrm{H}), 1.13-1.10(\mathrm{~m}, 36 \mathrm{H}), 0.86(\mathrm{~s}, 9 \mathrm{H}) ;{ }^{13} \mathrm{C} \mathrm{NMR}(125 \mathrm{MHz}$, $\left.\mathrm{CDCl}_{3}\right) \delta 109.1,47.8,32.6,30.7,27.9,23.8,18.3,12.0$; IR (thin film) 2945, 2867, 1465, 1255, $1061 \mathrm{~cm}^{-1}$; HRMS (ESI/Na) $m / z$ calcd for $\mathrm{C}_{28} \mathrm{H}_{60} \mathrm{O}_{4} \mathrm{Si}_{2} \mathrm{Na}(\mathrm{M}+\mathrm{Na})$ 539.3928, found 539.3917. Anal. Calcd for $\mathrm{C}_{28} \mathrm{H}_{60} \mathrm{O}_{4} \mathrm{Si}_{2}:$ C, 65.06; H, 11.70. Found: C, 65.52; H, 11.83 .
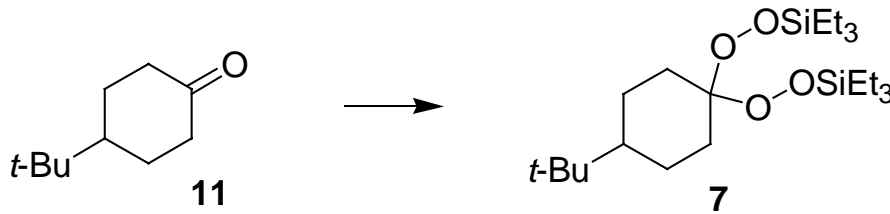

Triethylsilylperoxyketal (7). A solution of 4-tert-butylcyclohexanone $11(0.624 \mathrm{~g}, 4.05 \mathrm{mmol})$ in $6 \mathrm{~mL}$ of $\mathrm{CH}_{2} \mathrm{Cl}_{2}$ was treated with formic acid $(4.5 \mathrm{~mL}, 120 \mathrm{mmol}, 95-97 \%)$ and hydrogen peroxide $(7.2 \mathrm{~mL}, 120$ $\mathrm{mmol}, 50 \% \mathrm{H}_{2} \mathrm{O}$ ). The reaction mixture was vigorously stirred for $10 \mathrm{~min}$ at ambient temperature then added to $50 \mathrm{~mL} \mathrm{CH} \mathrm{Cl}_{2}$ and $50 \mathrm{~mL}$ of $\mathrm{H}_{2} \mathrm{O}$. The layers were separated and the aqueous layer was extracted with $3 \times 50$ $\mathrm{mL}$ of $\mathrm{CH}_{2} \mathrm{Cl}_{2}$. The organic layers were combined and the solvent was removed in vacuo to afford a white solid $(0.818 \mathrm{~g}, 99 \%)$. The unpurified product $(0.818 \mathrm{~g}, 4.00 \mathrm{mmol})$, in $10 \mathrm{~mL}$ of DMF was treated with $\mathrm{Et}_{3} \mathrm{~N}(1.41$ $\mathrm{mL}, 10.0 \mathrm{mmol}$ ), DMAP (0.0246 g, $0.200 \mathrm{mmol}$ in $2 \mathrm{~mL}$ of DMF) and $\mathrm{Et}_{3} \mathrm{SiOTf}(2.26 \mathrm{~mL}, 10.0 \mathrm{mmol})$. The reaction mixture was stirred for $18 \mathrm{~h}$ then added to $100 \mathrm{~mL}$ of ice-cold pentane $/ \mathrm{H}_{2} \mathrm{O}(1: 1)$. The organic layers were separated and the aqueous layer was extracted with $3 \times 30 \mathrm{~mL}$ of pentane. The organic layers were combined, dried over $\mathrm{Na}_{2} \mathrm{SO}_{4}$, filtered, and the solvent was removed in vacuo. Purification by flash chromatography (pentane) afforded 7 as a clear liquid $(1.30 \mathrm{~g}, 75 \%):{ }^{1} \mathrm{H} \mathrm{NMR}\left(500 \mathrm{MHz}, \mathrm{C}_{6} \mathrm{D}_{6}\right) \delta 2.61(\mathrm{~m}$, 
$2 \mathrm{H}), 1.56(\mathrm{~m}, 2 \mathrm{H}), 1.39(\mathrm{~m}, 4 \mathrm{H}), 1.15(\mathrm{t}, J=7.9,9 \mathrm{H}), 1.11(\mathrm{t}, J=7.9,9 \mathrm{H}), 0.87(\mathrm{~m}, 1 \mathrm{H}), 0.86(\mathrm{q}, J=8.0,6 \mathrm{H})$, $0.83(\mathrm{~s}, 9 \mathrm{H}), 0.80(\mathrm{q}, J=8.1,6 \mathrm{H}) ;{ }^{13} \mathrm{C}$ NMR $\left(125 \mathrm{MHz}, \mathrm{C}_{6} \mathrm{D}_{6}\right) \delta 109.9,48.1,32.7,31.4,28.1,24.4,7.53,4.80$, 4.76; IR (thin film) 2951, 2874, 1448, 1238, $1012 \mathrm{~cm}^{-1}$; HRMS $\left(\mathrm{CI} / \mathrm{NH}_{3}\right) \mathrm{m} / z$ calcd for $\mathrm{C}_{20} \mathrm{H}_{43} \mathrm{O}_{4} \mathrm{Si}_{2}(\mathrm{M}-\mathrm{Et})$ 403.2700, found 403.2702. Anal. Calcd for $\mathrm{C}_{22} \mathrm{H}_{48} \mathrm{O}_{4} \mathrm{Si}_{2}$ : C, 61.05; H, 11.18. Found: C, 61.23; H, 11.29.

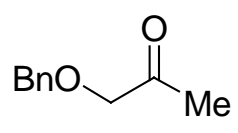

12

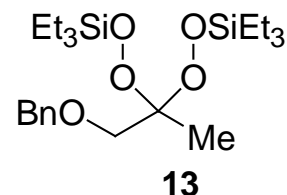

13

Triethylsilylperoxyketal (13). A solution of benzyloxyacetone $12(1.00 \mathrm{~g}, 6.10 \mathrm{mmol})$ in $20 \mathrm{~mL}$ of $\mathrm{CHCl}_{3}$ was treated with $\mathrm{H}_{2} \mathrm{O}_{2}\left(2.5 \mathrm{~mL}, 49 \mathrm{mmol}, 50 \% \mathrm{H}_{2} \mathrm{O}\right)$ and trifluoroacetic acid $(5.6 \mathrm{~mL}, 73 \mathrm{mmol}, 99 \%)$. The reaction mixture was stirred for $5 \mathrm{~min}$ at ambient temperature then sat. aq. $\mathrm{NaHCO}_{3}$ was added until the reaction mixture stopped bubbling. The reaction mixture was extracted with $6 \times 50 \mathrm{~mL}$ of $\mathrm{CH}_{2} \mathrm{Cl}_{2}$. The organic layers were combined and the solvent was removed in vacuo to afford a clear oil $\left(1.30 \mathrm{~g}, \sim 90 \%\right.$ product by ${ }^{1} \mathrm{H}$ NMR spectroscopy). The unpurified product $(1.30 \mathrm{~g}, 5.46 \mathrm{mmol})$ in $28 \mathrm{~mL}$ of DMF was treated with $\mathrm{Et}_{3} \mathrm{SiOTf}_{\mathrm{O}}$ $(3.94 \mathrm{~mL}, 17.5 \mathrm{mmol})$ and $\mathrm{Et}_{3} \mathrm{~N}(2.31 \mathrm{~mL}, 16.4 \mathrm{mmol})$. The reaction mixture was stirred for $4 \mathrm{~h}$ at room temperature then added to $100 \mathrm{~mL}$ of ice-cold pentane $/ \mathrm{H}_{2} \mathrm{O}(1: 1)$. The layers were separated and the aqueous layer was extracted with $4 \times 50 \mathrm{~mL}$ of pentane. The organic layers were combined, dried over $\mathrm{Na}_{2} \mathrm{SO}_{4}$, filtered, and the solvent was removed in vacuo. Purification by flash chromatography (100:0 - 60:40 pentane/EtOAc) afforded 13 as a clear liquid $(1.30 \mathrm{~g}, 54 \%):{ }^{1} \mathrm{H}$ NMR $\left(500 \mathrm{MHz}, \mathrm{CDCl}_{3}\right) \delta 7.35-7.23(\mathrm{~m}, 5 \mathrm{H}), 4.58(\mathrm{~s}, 2 \mathrm{H}), 3.69$ (s, 2H), $1.48(\mathrm{~s}, 3 \mathrm{H}), 0.97(\mathrm{~m}, 18 \mathrm{H}), 0.72(\mathrm{~m}, 12 \mathrm{H}) ;{ }^{13} \mathrm{C} \mathrm{NMR}\left(125 \mathrm{MHz}, \mathrm{CDCl}_{3}\right) \delta 138.7,128.5,127.69$, 127.65, 110.8, 73.6, 70.7, 17.5, 6.9, 4.0; IR (thin film) 2955, 2878, 1456, 1240, $1006 \mathrm{~cm}^{-1}$; LRMS (ESI/Na) $\mathrm{m} /$ $z$ calcd for $\mathrm{C}_{18} \mathrm{H}_{40} \mathrm{O}_{4} \mathrm{Si}_{2} \mathrm{Na}(\mathrm{M}+\mathrm{Na})$ 465.25, found 465.25. Anal. Calcd for $\mathrm{C}_{18} \mathrm{H}_{40} \mathrm{O}_{4} \mathrm{Si}_{2}: \mathrm{C}, 59.68 ; \mathrm{H}, 9.56$. Found: C, 66.32; H, 7.71.
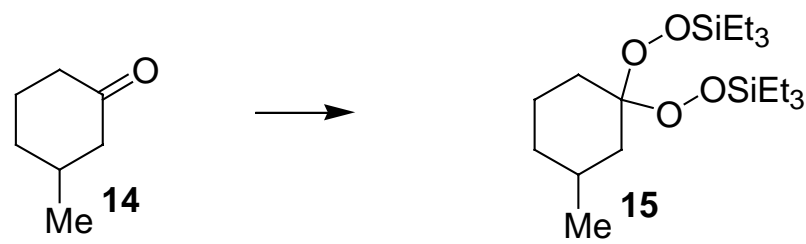

Triethylsilylperoxyketal (15). A solution of 3-methylcyclohexanone $\mathbf{1 4}(0.454 \mathrm{~g}, 4.05 \mathrm{mmol})$ in $6 \mathrm{~mL}$ of $\mathrm{CH}_{2} \mathrm{Cl}_{2}$ was treated with formic acid $(4.5 \mathrm{~mL}, 120 \mathrm{mmol}, 95-97 \%)$ and hydrogen peroxide $(7.2 \mathrm{~mL}, 120$ $\mathrm{mmol}, 50 \% \mathrm{H}_{2} \mathrm{O}$ ). The reaction mixture was stirred for $10 \mathrm{~min}$ at ambient temperature then added to $50 \mathrm{~mL}$ of $\mathrm{CH}_{2} \mathrm{Cl}_{2}$ and $50 \mathrm{~mL}$ of $\mathrm{H}_{2} \mathrm{O}$. The layers were separated and the aqueous layer was extracted with $3 \times 50 \mathrm{~mL}$ of $\mathrm{CH}_{2} \mathrm{Cl}_{2}$. The organic layers were combined and solvent was removed in vacuo to afford a clear oil $(0.455 \mathrm{~g}$, 95\% product by ${ }^{1} \mathrm{H}$ NMR spectroscopy). The unpurified product $(0.455 \mathrm{~g}, 2.81 \mathrm{mmol})$ in $12 \mathrm{~mL}$ of DMF was treated with $\mathrm{Et}_{3} \mathrm{~N}(1.79 \mathrm{~mL}, 12.7 \mathrm{mmol})$, DMAP $\left(17.3 \mathrm{mg}, 0.141 \mathrm{mmol}\right.$ in $2 \mathrm{~mL}$ of DMF) and $\mathrm{Et}_{3} \mathrm{SiOTf}(2.15$ $\mathrm{mL}, 12.7 \mathrm{mmol}$ ) at $0{ }^{\circ} \mathrm{C}$. The reaction mixture was stirred for $3 \mathrm{~h}$ from $0{ }^{\circ} \mathrm{C}-25{ }^{\circ} \mathrm{C}$ then added to $100 \mathrm{~mL}$ of ice-cold pentane $/ \mathrm{H}_{2} \mathrm{O}(1: 1)$. The layers were separated and the aqueous layer was extracted with $3 \times 30 \mathrm{~mL}$ of pentane. The organic layers were combined, dried over $\mathrm{Na}_{2} \mathrm{SO}_{4}$, filtered, and the solvent removed in vacuo. Purification by flash chromatography (pentane) afforded 15 as a clear liquid ( $0.857 \mathrm{~g}, 54 \%)$ : ${ }^{1} \mathrm{H}$ NMR (500 $\left.\mathrm{MHz}, \mathrm{CDCl}_{3}\right) \delta 2.25(\mathrm{~m}, 2 \mathrm{H}), 1.62(\mathrm{~m}, 3 \mathrm{H}), 1.47(\mathrm{~m}, J=13.4,3.6,1 \mathrm{H}), 1.22(\mathrm{~m}, J=13.5,4.4,1 \mathrm{H}), 0.99(\mathrm{~m}$, $18 \mathrm{H}), 0.94(\mathrm{~m}, 1 \mathrm{H}), 0.89(\mathrm{~d}, J=6.5,3 \mathrm{H}), 0.85(\mathrm{~m}, 1 \mathrm{H}), 0.71(\mathrm{~m}, 12 \mathrm{H}) ;{ }^{13} \mathrm{C} \mathrm{NMR}\left(125 \mathrm{MHz}, \mathrm{CDCl}_{3}\right) \delta 109.9$, 38.7, 34.7, 30.1, 29.4, 22.7, 22.4, 7.0, 4.2, 4.1; IR (thin film) 2954, 2878, 1459, 1240, $1006 \mathrm{~cm}^{-1}$; HRMS (ESI/Na) $m / z$ calcd for $\mathrm{C}_{19} \mathrm{H}_{42} \mathrm{O}_{4} \mathrm{Si}_{2} \mathrm{Na}(\mathrm{M}+\mathrm{Na}) 413.2519$, found 413.2520. Anal. Calcd for $\mathrm{C}_{19} \mathrm{H}_{42} \mathrm{O}_{4} \mathrm{Si}_{2}: \mathrm{C}$, 58.41; H, 10.84. Found: C, 58.69; H, 11.11. 


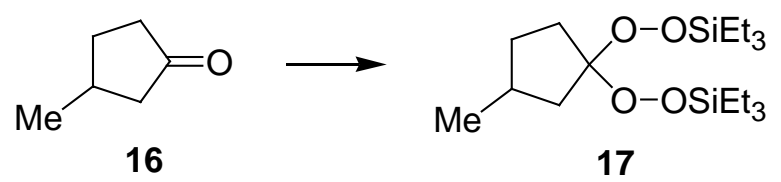

Triethylsilylperoxyketal (17). A solution of 3-methylcyclohexanone $16(0.398 \mathrm{~g}, 4.05 \mathrm{mmol})$ in $6 \mathrm{~mL}$ of $\mathrm{CH}_{2} \mathrm{Cl}_{2}$ was treated with formic acid $(4.5 \mathrm{~mL}, 120 \mathrm{mmol}, 95-97 \%)$ and hydrogen peroxide $(7.2 \mathrm{~mL}, 120$ $\mathrm{mmol}, 50 \% \mathrm{H}_{2} \mathrm{O}$ ). The reaction mixture was stirred for $10 \mathrm{~min}$ at ambient temperature then added to $50 \mathrm{~mL}$ of $\mathrm{CH}_{2} \mathrm{Cl}_{2}$ and $50 \mathrm{~mL}$ of $\mathrm{H}_{2} \mathrm{O}$. The layers were separated and the aqueous layer was extracted with $3 \times 50 \mathrm{~mL}$ of $\mathrm{CH}_{2} \mathrm{Cl}_{2}$. The organic layers were combined and the solvent was removed in vacuo to afford a clear oil $(0.479 \mathrm{~g}$, $\sim 95 \%$ product by ${ }^{1} \mathrm{H}$ NMR spectroscopy). The unpurified product $(0.479 \mathrm{~g}, 3.23 \mathrm{mmol})$ in $12 \mathrm{~mL}$ of DMF was treated with $\mathrm{Et}_{3} \mathrm{~N}$ (1.60 mL, $\left.11.3 \mathrm{mmol}\right)$, DMAP (0.0199 g, $0.162 \mathrm{mmol}$ in $2 \mathrm{~mL}$ of DMF) and $\mathrm{Et}_{3} \mathrm{SiOTf}_{\text {(2.56 }}$ $\mathrm{mL}, 11.3 \mathrm{mmol}$ ). The reaction mixture was stirred for $4 \mathrm{~h}$ at room temperature then added to $100 \mathrm{~mL}$ of ice-cold pentane $/ \mathrm{H}_{2} \mathrm{O}$ (1:1). The layers were separated and the aqueous layer was extracted with $3 \times 30 \mathrm{~mL}$ of pentane. The organic layers were combined, dried over $\mathrm{Na}_{2} \mathrm{SO}_{4}$, filtered, and the solvent was removed in vacuo. Purification by flash chromatography (pentane) $\mathbf{1 7}$ as a clear liquid $(0.783 \mathrm{~g}, 51 \%):{ }^{1} \mathrm{H}$ NMR $(500 \mathrm{MHz}$, $\left.\mathrm{CDCl}_{3}\right) \delta 2.18(\mathrm{~m}, 1 \mathrm{H}), 2.06(\mathrm{~m}, 2 \mathrm{H}), 1.92(\mathrm{~m}, 1 \mathrm{H}), 1.80(\mathrm{~m}, 1 \mathrm{H}), 1.49(\mathrm{dd}, J=10.0,3.9,1 \mathrm{H}), 1.29(\mathrm{~m}, 1 \mathrm{H})$, $1.02-0.98(\mathrm{~m}, 21 \mathrm{H}), 0.75-0.72(\mathrm{~m}, 12 \mathrm{H}) ;{ }^{13} \mathrm{C} \mathrm{NMR}\left(125 \mathrm{MHz}, \mathrm{CDCl}_{3}\right) \delta 120.9,42.0,33.67,33.55,33.47,20.0$, 7.0, 4.1; IR (thin film) 2956, 2878, 1459, 1240, $1006 \mathrm{~cm}^{-1}$; HRMS (ESI/Na) $m / z$ calcd for $\mathrm{C}_{18} \mathrm{H}_{40} \mathrm{O}_{4} \mathrm{Si}_{2} \mathrm{Na}(\mathrm{M}+$ Na) 399.2363, found 399.2379. Anal. Calcd for $\mathrm{C}_{18} \mathrm{H}_{40} \mathrm{O}_{4} \mathrm{Si}_{2}:$ C, 57.39; H, 10.70. Found: C, 57.56; H, 10.78.

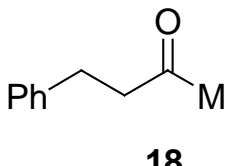

18

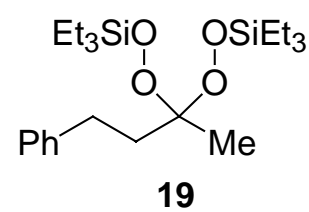

19

Triethylsilylperoxyketal (19). A solution of benzylacetone $18(0.600 \mathrm{~g}, 4.05 \mathrm{mmol})$ in $6 \mathrm{~mL}$ of $\mathrm{CH}_{2} \mathrm{Cl}_{2}$ was treated with formic acid $(4.5 \mathrm{~mL}, 120 \mathrm{mmol}, 95-97 \%)$ and hydrogen peroxide $(7.2 \mathrm{~mL}, 120 \mathrm{mmol}, 50 \%$ $\mathrm{H}_{2} \mathrm{O}$ ). The reaction mixture was stirred for $10 \mathrm{~min}$ at ambient temperature then added to $50 \mathrm{~mL}$ of $\mathrm{CH}_{2} \mathrm{Cl}_{2}$ and $50 \mathrm{~mL}$ of $\mathrm{H}_{2} \mathrm{O}$. The layers were separated and the aqueous layer was extracted $3 \times 50 \mathrm{~mL}$ with $\mathrm{CH}_{2} \mathrm{Cl}_{2}$. The organic layers were combined and the solvent was removed in vacuo to afford a clear oil $(0.685 \mathrm{~g}, \sim 1: 1$ mixture of peroxyketal and benzylacetone as detected by ${ }^{1} \mathrm{H}$ NMR spectroscopy). The unpurified product $(0.685 \mathrm{~g}$, $0.343 \mathrm{~g}$ of peroxyketal, $1.73 \mathrm{mmol})$, in $12 \mathrm{~mL}$ of DMF was treated with $\mathrm{Et}_{3} \mathrm{~N}(0.857 \mathrm{~mL}, 6.06 \mathrm{mmol})$, DMAP $\left(0.0106 \mathrm{~g}, 0.086 \mathrm{mmol}\right.$ in $2 \mathrm{~mL}$ of DMF) and $\mathrm{Et}_{3} \operatorname{SiOTf}(1.37 \mathrm{~mL}, 6.06 \mathrm{mmol})$ at $0{ }^{\circ} \mathrm{C}$. The reaction mixture was stirred for $3 \mathrm{~h}$ from $0{ }^{\circ} \mathrm{C}-25{ }^{\circ} \mathrm{C}$ then added to $100 \mathrm{~mL}$ of ice-cold pentane/ $\mathrm{H}_{2} \mathrm{O}$ (1:1). The layers were separated and the aqueous layer was extracted with $3 \times 30 \mathrm{~mL}$ of pentane. The organic layers were combined, dried over $\mathrm{Na}_{2} \mathrm{SO}_{4}$, filtered, and the solvent was removed in vacuo. Purification by flash chromatography (pentane) afforded 19 as a clear liquid $(0.857 \mathrm{~g}, 48 \%)$ : ${ }^{1} \mathrm{H}$ NMR $\left(500 \mathrm{MHz}, \mathrm{CDCl}_{3}\right) \delta 7.25(\mathrm{~m}, 3 \mathrm{H}), 7.18(\mathrm{~m}$, $2 \mathrm{H}), 2.70(\mathrm{~m}, 2 \mathrm{H}), 2.03(\mathrm{~m}, 2 \mathrm{H}), 1.41(\mathrm{~s}, 3 \mathrm{H}), 0.99(\mathrm{t}, J=4.6,18 \mathrm{H}), 0.71(\mathrm{q}, J=7.9,12 \mathrm{H}) ;{ }^{13} \mathrm{C}$ NMR $(125$ $\left.\mathrm{MHz}, \mathrm{CDCl}_{3}\right) \delta 142.5,128.59,128.58,126.0,110.7,36.3,30.9,19.1,7.0,4.1$; IR (thin film) $2950,2880,1458$, 1234, $1009 \mathrm{~cm}^{-1}$; HRMS (CI/ $\left.\mathrm{NH}_{3}\right) \mathrm{m} / z$ calcd for $\mathrm{C}_{22} \mathrm{H}_{46} \mathrm{O}_{4} \mathrm{Si}_{2} \mathrm{~N}$ (M) 444.2965, found 444.2964. Anal. Calcd for $\mathrm{C}_{22} \mathrm{H}_{42} \mathrm{O}_{4} \mathrm{Si}_{2}: \mathrm{C}, 61.92 ; \mathrm{H}, 9.92$. Found: $\mathrm{C}, 62.02 ; \mathrm{H}, 10.02$. 


\section{Preparation of Alkenes}

Alkenes $\mathbf{4}^{6}, \mathbf{2 8}^{7}$, and $\mathbf{2 6}^{8}$ were prepared according to literature procedures (see bibliography).<smiles>CS(Cl)(Cl)c1ccccc1</smiles><smiles>C=C(C)C[Si](C)(C)C(c1ccccc1)c1ccccc1</smiles>

Benzhydryldimethyl-(2-methylallyl)silane (30a). Magnesium metal (0.930 g, $38.3 \mathrm{mmol}, 50 \mathrm{mesh})$ was stirred vigorously for $16 \mathrm{~h}$ under $\mathrm{N}_{2}$ then $17 \mathrm{~mL}$ of THF were added. The resultant gray suspension was treated with methallylchloride $(0.900 \mathrm{~mL}, 9.20 \mathrm{mmol})$ dropwise at $0{ }^{\circ} \mathrm{C}-25^{\circ} \mathrm{C}$. After $1 \mathrm{~h}$, the reaction mixture was cooled to $0{ }^{\circ} \mathrm{C}$ and benzhydryldimethylchlorosilane ${ }^{7}(2.00 \mathrm{~g}, 7.67 \mathrm{mmol})$ was added dropwise. The reaction mixture was allowed to warm to ambient temperature and stirred for 3 days, followed by addition of $\mathrm{HCl}(1 \mathrm{M})$ at $0{ }^{\circ} \mathrm{C}$. The reaction mixture was extracted with $3 \times 100 \mathrm{~mL}$ of $\mathrm{Et}_{2} \mathrm{O}$. The organic layers were combined, dried over $\mathrm{Na}_{2} \mathrm{SO}_{4}$, filtered, and the solvent was removed in vacuo. Purification by flash chromatography (100:0 - 90:10 hexanes/EtOAc) afforded 30a as a colorless oil (1.63 g, 76\%): ${ }^{1} \mathrm{H} \mathrm{NMR}(500$ $\left.\mathrm{MHz}, \mathrm{CDCl}_{3}\right) \delta 7.24(\mathrm{~m}, 8 \mathrm{H}), 7.13(\mathrm{~m}, 2 \mathrm{H}), 4.57(\mathrm{~s}, 1 \mathrm{H}), 4.39(\mathrm{~s}, 1 \mathrm{H}), 3.57(\mathrm{~s}, 1 \mathrm{H}), 1.63(\mathrm{~s}, 3 \mathrm{H}), 1.54(\mathrm{~s}, 2 \mathrm{H})$, $0.07(\mathrm{~s}, 6 \mathrm{H}) ;{ }^{13} \mathrm{C}$ NMR $\left(125 \mathrm{MHz}, \mathrm{CDCl}_{3}\right) \delta 143.3,142.8,129.0,128.6,128.5,125.3,109.2,45.4,26.3,25.4,-$ 3.0; IR (thin film) 3066, 2955, 1633, 1488, $1244 \mathrm{~cm}^{-1}$; HRMS (EI) $m / z$ calcd for $\mathrm{C}_{19} \mathrm{H}_{24} \mathrm{Si}(\mathrm{M}) 280.1647$, found 280.1643. Anal. Calcd for $\mathrm{C}_{19} \mathrm{H}_{24} \mathrm{Si}$ : C, 81.36; H, 8.62. Found: C, 81.34; H, 8.70.<smiles>C/C(=C/C[Si](C)(C)C(C)c1ccccc1)c1ccccc1</smiles>

$30 a$
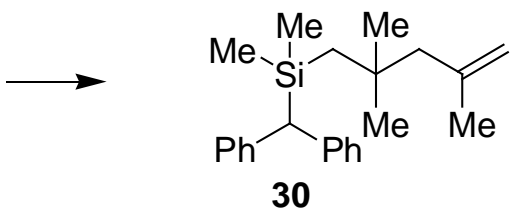

Benzhydryldimethyl-(2,2,4-trimethylpent-4-enyl)silane (30). A solution of benzhydryldimethyl-(2methylallyl)silane 30a $(0.100 \mathrm{~g}, 0.402 \mathrm{mmol})$ in $17 \mathrm{~mL}$ of $\mathrm{CH}_{2} \mathrm{Cl}_{2}$ was treated with $\mathrm{SnCl}_{4}(0.4 \mathrm{~mL}, 1 \mathrm{M}$ in $\mathrm{CH}_{2} \mathrm{Cl}_{2}$ ) at $-78{ }^{\circ} \mathrm{C}$. The reaction mixture was allowed to warm to $-3{ }^{\circ} \mathrm{C}$ over $22 \mathrm{~h}$ then added to $10 \mathrm{~mL}$ of icecold $\mathrm{H}_{2} \mathrm{O}$. The reaction mixture was extracted with $3 \times 50 \mathrm{~mL}$ of $\mathrm{CH}_{2} \mathrm{Cl}_{2}$. The organic layers were combined, dried over $\mathrm{Na}_{2} \mathrm{SO}_{4}$, filtered, and the solvent was removed in vacuo. Purification by flash chromatography (100:0 - 90:10 hexanes/EtOAc) afforded 30 as a clear oil $(0.035 \mathrm{~g}, 52 \%):{ }^{1} \mathrm{H}$ NMR $\left(500 \mathrm{MHz}, \mathrm{CDCl}_{3}\right) \delta 7.26$ $(\mathrm{m}, 8 \mathrm{H}), 7.15(\mathrm{~m}, 2 \mathrm{H}), 4.82(\mathrm{~s}, 1 \mathrm{H}), 4.56(\mathrm{~s}, 1 \mathrm{H}), 3.50(\mathrm{~s}, 1 \mathrm{H}), 1.92(\mathrm{~s}, 2 \mathrm{H}), 1.66(\mathrm{~s}, 3 \mathrm{H}), 0.95(\mathrm{~s}, 6 \mathrm{H}), 0.74(\mathrm{~s}$, $2 \mathrm{H}), 0.16(\mathrm{~s}, 6 \mathrm{H}) ;{ }^{13} \mathrm{C} \mathrm{NMR}\left(125 \mathrm{MHz}, \mathrm{CDCl}_{3}\right) \delta 146.9,146.1,131.9,131.3,128.1,117.2,56.7,49.9,37.5$, 33.4, 33.1, 28.5, 2.6; IR (thin film) 3061, 2913, 1640, 1494, $1249 \mathrm{~cm}^{-1}$; LRMS (ESI/Na) $\mathrm{m} / \mathrm{z}$ calcd for $\mathrm{C}_{23} \mathrm{H}_{33} \mathrm{Si}(\mathrm{M}+\mathrm{H})$ 336.24, found 337.50. Anal. Calcd for $\mathrm{C}_{23} \mathrm{H}_{32} \mathrm{Si}$ : C, 82.07; H, 9.58. Found: C, 82.03; $\mathrm{H}, 9.73$.

\section{Annulation Products Between Peroxycarbenium ions and Alkenes}

General Procedure for the Annulation of Peroxycarbenium ions with Alkenes. Unless otherwise noted a solution of silyl peroxyketal (1.0 equiv) and alkene (3.0 equiv) in $\mathrm{CH}_{2} \mathrm{Cl}_{2}(0.16 \mathrm{M})$ was treated with $\mathrm{SnCl}_{4}$ (2.0 equiv, $1.0 \mathrm{M}$ solution in $\mathrm{CH}_{2} \mathrm{Cl}_{2}$ ) at $-78{ }^{\circ} \mathrm{C}$. The reaction mixture was allowed to warm to $-3{ }^{\circ} \mathrm{C}$ over $2 \mathrm{~h}-24 \mathrm{~h}$ then added to ice-cold $\mathrm{H}_{2} \mathrm{O}$. The reaction mixture was extracted with $\mathrm{CH}_{2} \mathrm{Cl}_{2}$. The organic layers were combined, dried over $\mathrm{Na}_{2} \mathrm{SO}_{4}$, filtered, and concentrated in vacuo. Purification by flash chromatography was performed using Davisil ${ }^{\circledR}$ silica gel. 


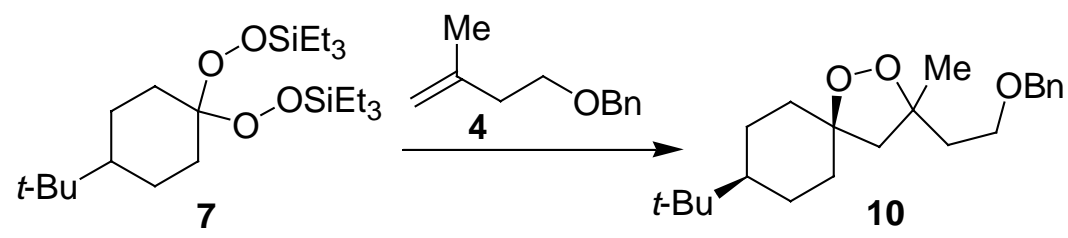

3-(2-Benzyloxyethyl)-8-tert-butyl-3-methyl-1,2-dioxaspiro[4.5]decane (10). The standard annulation procedure was followed with silyl peroxyketal $7(0.050 \mathrm{~g}, 0.116 \mathrm{mmol})$ and 2-methyl-4-(phenylmethoxy)-1butene $4(0.0613 \mathrm{~g}, 0.348 \mathrm{mmol})$ in $5 \mathrm{~mL}$ of $\mathrm{CH}_{2} \mathrm{Cl}_{2}$ and $\mathrm{SnCl}_{4}(0.23 \mathrm{~mL})$. Purification by flash chromatography (100:0 - 60:40 hexanes/EtOAc) afforded 10 as a clear liquid $(0.0320 \mathrm{~g}, 80 \%):{ }^{1} \mathrm{H}$ NMR (500 $\left.\mathrm{MHz} \mathrm{CDCl}_{3}\right) \delta 7.32(\mathrm{~m}, 5 \mathrm{H}), 4.51(\mathrm{~d}, J=11.9,1 \mathrm{H}), 4.47(\mathrm{~d}, J=11.9,1 \mathrm{H}), 3.59(\mathrm{~m}, 2 \mathrm{H}), 2.22(\mathrm{~d}, J=12.0$, $1 \mathrm{H}), 2.01(\mathrm{~m}, 4 \mathrm{H}), 1.91(\mathrm{~m}, 1 \mathrm{H}), 1.58(\mathrm{~m}, 2 \mathrm{H}), 1.40(\mathrm{~m}, 2 \mathrm{H}), 1.32(\mathrm{~s}, 3 \mathrm{H}), 1.29(\mathrm{~m}, 2 \mathrm{H}), 0.95(\mathrm{~m}, 1 \mathrm{H}), 0.83(\mathrm{~s}$, 9H); ${ }^{13} \mathrm{C}$ NMR $\left(125 \mathrm{MHz}, \mathrm{CDCl}_{3}\right) \delta 138.3,128.4,127.7,127.6,84.5,84.2,73.1,66.8,57.6,47.1,38.9,36.1$, 35.9, 32.4, 27.5, 24.4, 23.8, 23.7; IR (thin film) 3031, 2941, 1454, $1110 \mathrm{~cm}^{-1}$; HRMS (ESI/Na) $\mathrm{m} / \mathrm{z}$ calcd for $\mathrm{C}_{22} \mathrm{H}_{34} \mathrm{O}_{3} \mathrm{Na}(\mathrm{M}+\mathrm{Na})$ 369.2406, found 369.2412. Anal. Calcd for $\mathrm{C}_{22} \mathrm{H}_{34} \mathrm{O}_{3}$ : C, 76.26; H, 9.89. Found: C, $76.50 ; \mathrm{H}, 9.92$.
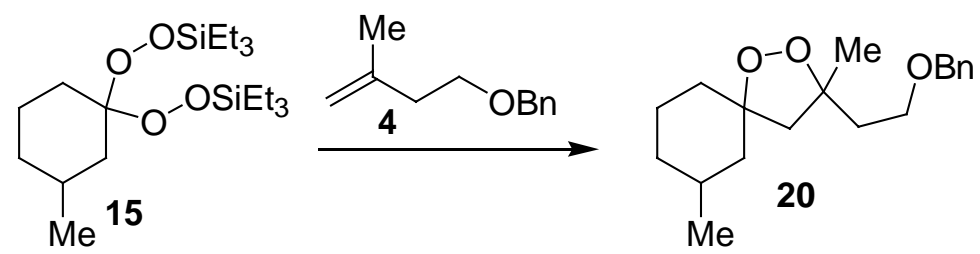

3-(2-Benzyloxyethyl)-3,7-dimethyl-1,2-dioxaspiro[4.5]decane (20). The standard annulation procedure was followed with silyl peroxyketal $15(0.050 \mathrm{~g}, 0.128 \mathrm{mmol})$ and 2-methyl-4-(phenylmethoxy)-1butene $4(0.068 \mathrm{~g}, 0.384 \mathrm{mmol})$ in $5 \mathrm{~mL}$ of $\mathrm{CH}_{2} \mathrm{Cl}_{2}$ and $\mathrm{SnCl}_{4}(0.26 \mathrm{~mL})$. Purification by flash chromatography (100:0 - 60:40 pentane/EtOAc) afforded 20 as a clear liquid $\left(0.0280 \mathrm{~g}, 72 \%\right.$, d.r. 1:1): ${ }^{1} \mathrm{H}$ NMR $(500 \mathrm{MHz}$, $\left.\mathrm{CDCl}_{3}\right) \delta 7.32(\mathrm{~m}, 8 \mathrm{H}), 7.28(\mathrm{~m}, 2 \mathrm{H}), 4.50(\mathrm{~m}, 4 \mathrm{H}), 3.61(\mathrm{~m}, 4 \mathrm{H}), 2.25(\mathrm{t}, J=12.3,2 \mathrm{H}), 2.06-1.91(\mathrm{~m}, 10 \mathrm{H})$, $1.67(\mathrm{~m}, 4 \mathrm{H}), 1.56(\mathrm{~m}, 4 \mathrm{H}), 1.34(\mathrm{~s}, 3 \mathrm{H}), 1.33(\mathrm{~s}, 3 \mathrm{H}), 1.29(\mathrm{~m}, 2 \mathrm{H}), 1.06(\mathrm{~m}, 2 \mathrm{H}), 0.87(\mathrm{~d}, J=5.3,3 \mathrm{H}), 0.86$ $(\mathrm{d}, J=5.2,3 \mathrm{H}), 0.80(\mathrm{~m}, 2 \mathrm{H}) ;{ }^{13} \mathrm{C}$ NMR $\left(125 \mathrm{MHz}, \mathrm{CDCl}_{3}\right) \delta 138.6,138.5,128.6,127.86,127.77,85.54$, 85.53, 84.37, 84.36, 73.3, 67.0, 66.99, 58.37, 58.29, 44.5, 44.3, 39.2, 39.0, 35.4, 35.3, 34.1, 29.34, 29.28, 24.8, 24.5, 22.9, 22.8, 22.73, 22.72; IR (thin film) 3031, 2926, 2865, $1454 \mathrm{~cm}^{-1}$; HRMS (ESI/Na) $\mathrm{m} / z$ calcd for $\mathrm{C}_{19} \mathrm{H}_{28} \mathrm{O}_{3} \mathrm{Na}(\mathrm{M}+\mathrm{Na})$ 327.1936, found 327.1937. Anal. Calcd for $\mathrm{C}_{19} \mathrm{H}_{28} \mathrm{O}_{3}: \mathrm{C}, 74.96 ; \mathrm{H}, 9.27$. Found: $\mathrm{C}$, 74.98; H, 9.47.
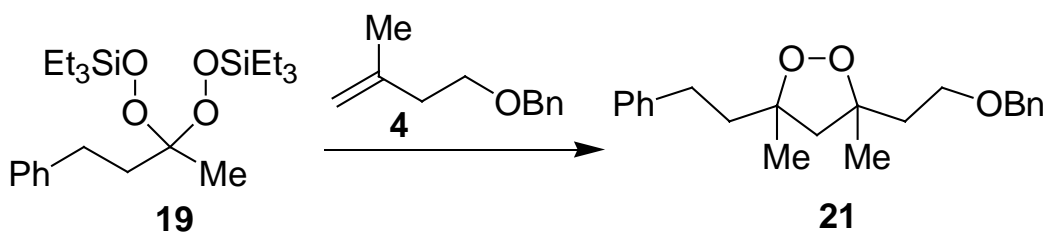

3-(2-Benzyloxyethyl)-3,5-dimethyl-5-phenethyl[1.2]dioxolane (21). The standard annulation procedure was followed with silyl peroxyketal $19(0.50 \mathrm{~g}, 0.114 \mathrm{mmol})$ and 2-methyl-4-(phenylmethoxy)-1butene $4(0.060 \mathrm{mg}, 0.342 \mathrm{mmol})$ in $5 \mathrm{~mL}$ of $\mathrm{CH}_{2} \mathrm{Cl}_{2}$ and $\mathrm{SnCl}_{4}(0.23 \mathrm{~mL})$. Purification by flash chromatography (100:0 - 60:40 pentane/EtOAc) afforded 21 as a clear liquid $\left(0.0224\right.$ g, 57\%, d.r. 1:1): ${ }^{1} \mathrm{H}$ $\operatorname{NMR}\left(500 \mathrm{MHz}, \mathrm{CDCl}_{3}\right) \delta 7.36(\mathrm{~m}, 8 \mathrm{H}), 7.30(\mathrm{~m}, 6 \mathrm{H}), 7.21(\mathrm{~m}, 6 \mathrm{H}), 4.52(\mathrm{~s}, 2 \mathrm{H}), 4.50(\mathrm{~s}, 2 \mathrm{H}), 3.62(\mathrm{~m}, 4 \mathrm{H})$, 
$2.71(\mathrm{~m}, 4 \mathrm{H}), 2.44(\mathrm{~d}, J=12.0,1 \mathrm{H}), 2.35(\mathrm{~d}, J=12.1,1 \mathrm{H}), 2.22(\mathrm{~d}, J=12.1,1 \mathrm{H}), 2.13(\mathrm{~d}, J=12.1,1 \mathrm{H}), 2.00$ $(\mathrm{m}, 6 \mathrm{H}), 1.85(\mathrm{~m}, 2 \mathrm{H}), 1.42(\mathrm{~s}, 3 \mathrm{H}), 1.389(\mathrm{~s}, 3 \mathrm{H}), 1.386(\mathrm{~s}, 3 \mathrm{H}), 1.35(\mathrm{~s}, 3 \mathrm{H}) ;{ }^{13} \mathrm{C}$ NMR $\left(125 \mathrm{MHz}, \mathrm{CDCl}_{3}\right) \delta$ $142.26,142.20,138.52$, 138.50, 128.64, 128.63, 128.61, 128.59, 128.50, 128.49, 127.87, 127.84, 127.81, $127.79,126.1,85.9,85.2,73.35,73.32,66.95,66.94,56.9,56.8,41.7,41.4,39.14,39.06,31.18,31.13,24.7$, 24.5, 24.3, 24.0; IR (thin film) 3028, 2933, 2865, $1497 \mathrm{~cm}^{-1}$; HRMS (ESI/Na) $\mathrm{m} / z$ calcd for $\mathrm{C}_{22} \mathrm{H}_{28} \mathrm{O}_{3} \mathrm{Na}(\mathrm{M}+$ Na) 363.1936, found 363.1945. Anal. Calcd for $\mathrm{C}_{22} \mathrm{H}_{28} \mathrm{O}_{3}$ : C, 77.61; H, 8.29. Found: C, 76.36; H, 8.27.
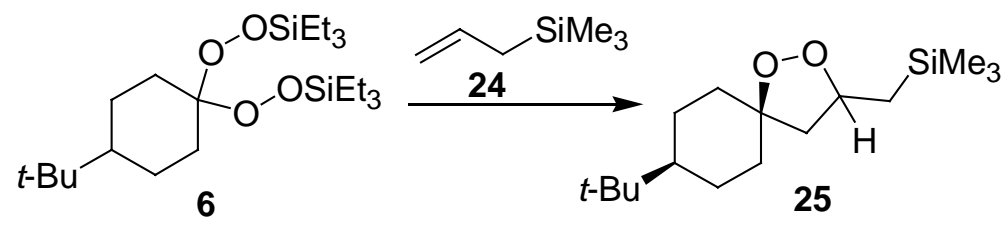

(8-tert-Butyl-1,2-dioxaspiro[4.5]dec-3-ylmethyl)-trimethylsilane (25). ${ }^{9}$ To a $-78{ }^{\circ} \mathrm{C}$ solution of silyl peroxyketal $6(0.500 \mathrm{~g}, 1.16 \mathrm{mmol})$ and $40 \mathrm{~mL}$ of $\mathrm{CH}_{2} \mathrm{Cl}_{2}$, was added allyltrimethylsilane $\mathbf{2 4}(1.88 \mathrm{~mL}, 11.6$ mmol). After $5 \mathrm{~min}, \mathrm{SnCl}_{4}(4.6 \mathrm{~mL})$ was slowly added to the reaction mixture. The reaction mixture was stirred for $24 \mathrm{~h}$ from $-78{ }^{\circ} \mathrm{C}$ to ambient temperature then $50 \mathrm{~mL}$ of $\mathrm{H}_{2} \mathrm{O}$ were added. The reaction mixture was extracted with $3 \times 20 \mathrm{~mL}$ of $\mathrm{CH}_{2} \mathrm{Cl}_{2}$ and the combined organic layers were concentrated in vacuo. Purification by flash chromatography $\left(80: 20\right.$ hexanes/ $\left.\mathrm{CH}_{2} \mathrm{Cl}_{2}\right)$ afforded $\mathbf{2 5}$ as a white solid $(0.30 \mathrm{~g}, 92 \%)$ : $\mathrm{mp} 73.0-74.0{ }^{\circ} \mathrm{C}$; ${ }^{1} \mathrm{H}$ NMR $\left(500 \mathrm{MHz}, \mathrm{CDCl}_{3}\right) \delta 4.33(\mathrm{~m}, 1 \mathrm{H}), 2.32(\mathrm{dd}, J=11.6,6.9,1 \mathrm{H}), 2.04(\mathrm{~m}, J=9.8,2.9,1 \mathrm{H}), 1.95(\mathrm{~m}, J$ $=10.5,3.1,1 \mathrm{H}), 1.81(\mathrm{dd}, J=11.6,8.1,1 \mathrm{H}), 1.55(\mathrm{~m}, 4 \mathrm{H}), 1.36(\mathrm{~m}, 2 \mathrm{H}), 1.21(\mathrm{~m}, J=13.4,3.7,1 \mathrm{H}), 1.07(\mathrm{dd}$, $J=14.0,5.5,1 \mathrm{H}), 0.95(\mathrm{~m}, 1 \mathrm{H}), 0.9-0.8(\mathrm{~m}, 1 \mathrm{H}), 0.84(\mathrm{~s}, 9 \mathrm{H}), 0.05(\mathrm{~s}, 9 \mathrm{H}) ;{ }^{13} \mathrm{C}$ NMR $\left(125 \mathrm{MHz}, \mathrm{CDCl}_{3}\right) \delta$ 84.3, 79.4, 54.4, 47.2, 36.8, 36.2, 32.4, 27.6, 23.81, 23.78, 21.1, -1.0; IR (KBr) 2952, 1361, 1251, 861, $840 \mathrm{~cm}$ ${ }^{1}$; HRMS (CI/NH $\mathrm{NH}_{3} \mathrm{~m} / \mathrm{z}$ calcd for $\mathrm{C}_{16} \mathrm{H}_{32} \mathrm{O}_{2} \mathrm{Si}$ (M) 284.2172, found 284.2166. Anal. Calcd for $\mathrm{C}_{16} \mathrm{H}_{32} \mathrm{O}_{2} \mathrm{Si}$ : C, 67.54; H, 11.34. Found: C, 67.75; H, 11.34.
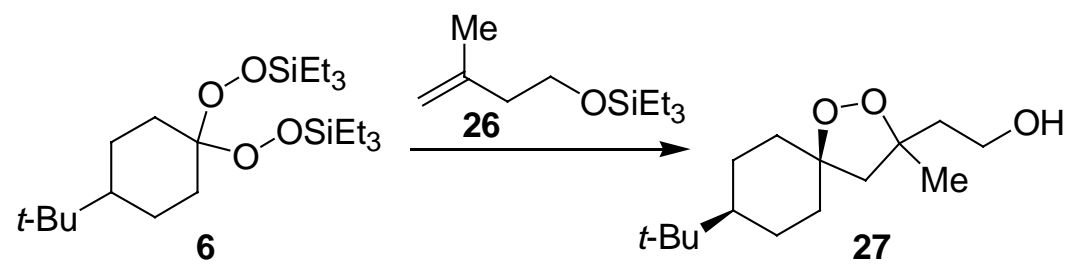

2-(8-tert-Butyl-1,2-dioxaspiro[4.5]dec-3-yl)-ethanol (27). The standard annulation procedure was followed with silyl peroxyketal $6(0.0350 \mathrm{~g}, 0.0809 \mathrm{mmol})$ and triethyl-(3-methyl-but-3-enyloxy)-silane $\mathbf{2 6}(0.0490 \mathrm{~g}$, $0.243 \mathrm{mmol})$ in $3.5 \mathrm{~mL}$ of $\mathrm{CH}_{2} \mathrm{Cl}_{2}$ and $\mathrm{SnCl}_{4}(0.16 \mathrm{~mL})$. Purification by flash chromatography (100:0 - 50:50 pentane/EtOAc) afforded 27 as a clear liquid $(0.0159 \mathrm{~g}, 77 \%):{ }^{1} \mathrm{H}$ NMR $\left(500 \mathrm{MHz}, \mathrm{CDCl}_{3}\right) \delta 3.78(\mathrm{~m}, 2 \mathrm{H})$, $2.22(\mathrm{~d}, J=12,1 \mathrm{H}), 2.09(\mathrm{~m}, 2 \mathrm{H}), 1.99(\mathrm{~m}, 2 \mathrm{H}), 1.81(\mathrm{ddd}, J=14.4,5.5,6.6,2 \mathrm{H}), 1.61(\mathrm{~m}, 2 \mathrm{H}), 1.48(\mathrm{~m}, J=$ 13.7, 4.1, 1H), $1.39(\mathrm{~m}, J=13.6,3.7,1 \mathrm{H}), 1.36(\mathrm{~s}, 3 \mathrm{H}), 1.31(\mathrm{~m}, 2 \mathrm{H}), 0.96(\mathrm{~m}, 1 \mathrm{H}), 0.88(\mathrm{~m}, 1 \mathrm{H}), 0.83(\mathrm{~s}, 9 \mathrm{H})$; ${ }^{13} \mathrm{C}$ NMR $\left(125 \mathrm{MHz}, \mathrm{CDCl}_{3}\right) \delta 85.3,84.8,59.3,57.6,47.1,41.4,36.1,35.7,32.4,27.5,24.4,23.9,23.7 \mathrm{~cm}^{-1}$; IR (thin film) 3339, 2942, 2868, $1442 \mathrm{~cm}^{-1}$; HRMS (ESI/Na) $\mathrm{m} / z$ calcd for $\mathrm{C}_{15} \mathrm{H}_{28} \mathrm{O}_{3} \mathrm{Na}(\mathrm{M}+\mathrm{Na}) 279.1936$, found 279.1933. Anal. Calcd for $\mathrm{C}_{15} \mathrm{H}_{28} \mathrm{O}_{3}: \mathrm{C}, 70.27 ; \mathrm{H}, 11.01$. Found: $\mathrm{C}, 69.59 ; \mathrm{H}, 11.09$.

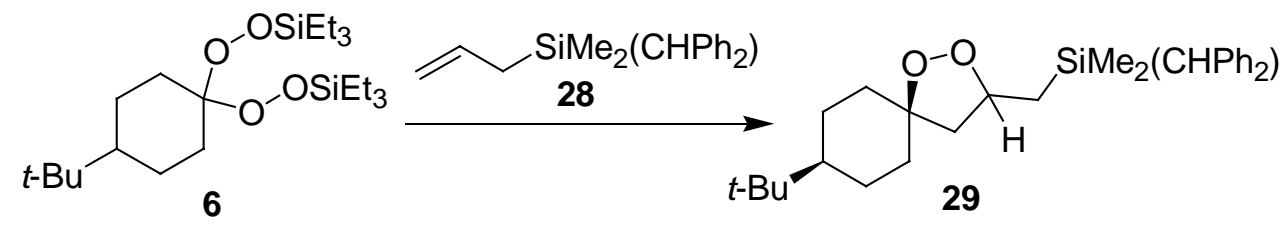


Benzhydryl-(8-tert-butyl-1,2-dioxaspiro[4.5]dec-3-ylmethyl)-dimethylsilane (29). To a - $78{ }^{\circ} \mathrm{C}$ solution of silyl peroxyketal $6(2.0 \mathrm{~g}, 4.6 \mathrm{mmol})$ and $200 \mathrm{~mL}$ of $\mathrm{CH}_{2} \mathrm{Cl}_{2}$, was added benzhydryldimethylsilane $28(3.7 \mathrm{~g}, 13.9 \mathrm{mmol})$. After $5 \mathrm{~min}, \mathrm{SnCl}_{4}(4.6 \mathrm{~mL})$ was slowly added to the reaction mixture. The reaction mixture was stirred for $22 \mathrm{~h}$ from $-78{ }^{\circ} \mathrm{C}$ to ambient temperature then $100 \mathrm{~mL} \mathrm{of} \mathrm{H}_{2} \mathrm{O}$ were added. The reaction mixture was extracted with $3 \times 200 \mathrm{~mL}$ of $\mathrm{CH}_{2} \mathrm{Cl}_{2}$ and the combined organic layers were concentrated in vacuo. Purification by flash chromatography (80:20 hexanes/ $\left.\mathrm{CH}_{2} \mathrm{Cl}_{2}\right)$ afforded 29 as a white solid (1.4 g, 72\%): $\mathrm{mp}$ 86.0-87.0 ${ }^{\circ} \mathrm{C}$; ${ }^{1} \mathrm{H}$ NMR $\left(500 \mathrm{MHz}, \mathrm{CDCl}_{3}\right) \delta 7.25(\mathrm{~m}, 8 \mathrm{H}), 7.14(\mathrm{~m}, 2 \mathrm{H}) 4.21(\mathrm{~m}, 1 \mathrm{H}), 3.57(\mathrm{~s}, 1 \mathrm{H})$, $2.21(\mathrm{dd}, J=6.9,4.7,1 \mathrm{H}), 1.94(\mathrm{~m}, 1 \mathrm{H}), 1.88(\mathrm{~m}, 1 \mathrm{H}), 1.69(\mathrm{dd}, J=7.8,3.8,1 \mathrm{H}), 1.58(\mathrm{~m}, 2 \mathrm{H}), 1.44(\mathrm{~m}, J=$ $14,4.4,1 \mathrm{H}), 1.33-1.25(\mathrm{~m}, 3 \mathrm{H}), 1.17(\mathrm{~m}, 1 \mathrm{H}), 1.05(\mathrm{dd}, J=14,6.6,1 \mathrm{H}), 0.93(\mathrm{~m}, 1 \mathrm{H}), 0.89(\mathrm{~s}, 9 \mathrm{H}), 0.10(\mathrm{~s}$, $3 \mathrm{H}), 0.096(\mathrm{~s}, 3 \mathrm{H}) ;{ }^{13} \mathrm{C}$ NMR $\left(125 \mathrm{MHz}, \mathrm{CDCl}_{3}\right) \delta 142.3,128.8,128.7,128.44,128.40,125.4,125.3,84.3$, 79.0, 54.5, 47.2, 45.3, 36.6, 36.1, 32.4, 27.5, 24.8, 23.7, 19.4, -2.5, -2.6; IR (thin film) 3058, 2867, 1596, 1494, $1248 \mathrm{~cm}^{-1}$; HRMS $\left(\mathrm{CI} / \mathrm{NH}_{3}\right) \mathrm{m} / z$ calcd for $\mathrm{C}_{28} \mathrm{H}_{41} \mathrm{O}_{2} \mathrm{Si}(\mathrm{M}+\mathrm{H})$ 437.2876, found 437.2876. Anal. Calcd for $\mathrm{C}_{28} \mathrm{H}_{40} \mathrm{O}_{2} \mathrm{Si}: \mathrm{C}, 77.01 ; \mathrm{H}, 9.23$. Found: C, 77.22; H, 9.23.
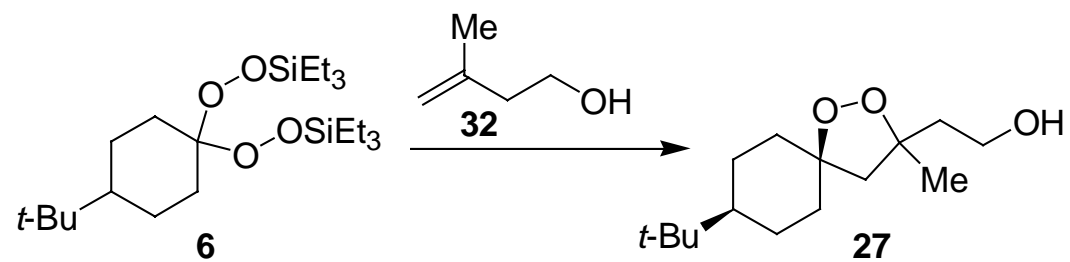

2-(8-tert-Butyl-1,2-dioxaspiro[4.5]dec-3-yl)-ethanol (27). The standard annulation procedure was followed with silyl peroxyketal $27(0.300 \mathrm{~g}, 0.696 \mathrm{mmol})$ and 3-Methyl-but-3-en-1-ol $32(0.180 \mathrm{~g}, 2.09 \mathrm{mmol})$ in $30 \mathrm{~mL}$ of $\mathrm{CH}_{2} \mathrm{Cl}_{2}$ and $\mathrm{SnCl}_{4}(1.4 \mathrm{~mL})$. Purification by flash chromatography (100:0 - 50:50 pentane/EtOAc) afforded 27 as a clear liquid $(0.0840 \mathrm{~g}, 47 \%)$ : Characterization data matched.

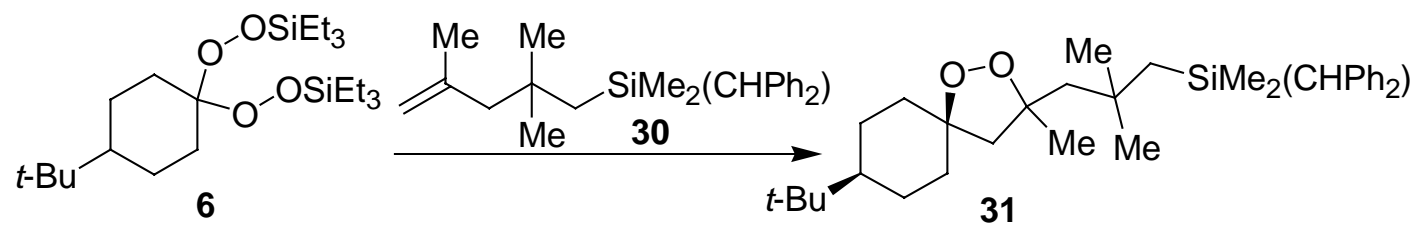

Benzhydryl-[3-(8-tert-butyl-3-methyl-1,2-dioxaspiro[4.5]dec-3-yl)-2,2-dimethylpropyl]dimethylsilane (31). The standard annulation procedure was followed with silyl peroxyketal $6(0.0140 \mathrm{~g}, 0.0324 \mathrm{mmol})$

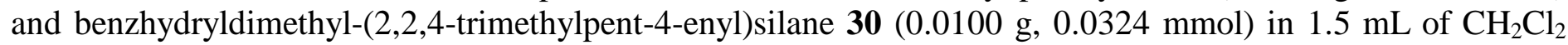
and $\mathrm{SnCl}_{4}(0.032 \mathrm{~mL})$. Purification by flash chromatography $(100: 0-80: 20$ pentane/EtOAc) afforded 31 as a clear liquid $(0.0090 \mathrm{~g}, 55 \%):{ }^{1} \mathrm{H}$ NMR $\left(500 \mathrm{MHz}, \mathrm{CDCl}_{3}\right) \delta 7.23(\mathrm{~m}, 8 \mathrm{H}), 7.12(\mathrm{~m}, 2 \mathrm{H}), 3.48(\mathrm{~s}, 1 \mathrm{H}), 1.96(\mathrm{~m}$, 4H), $1.69(\mathrm{~d}, J=14.4,1 \mathrm{H}), 1.59(\mathrm{~m}, 2 \mathrm{H}), 1.46(\mathrm{~m}, 2 \mathrm{H}), 1.44(\mathrm{~m}, J=13.6,4.0,1 \mathrm{H}), 1.34(\mathrm{~m}, 1 \mathrm{H}), 1.27(\mathrm{~s}, 3 \mathrm{H})$, $1.24(\mathrm{~m}, 1 \mathrm{H}), 0.997(\mathrm{~s}, 3 \mathrm{H}), 0.994(\mathrm{~s}, 3 \mathrm{H}), 0.92(\mathrm{~m}, 1 \mathrm{H}), 0.83(\mathrm{~s}, 9 \mathrm{H}), 0.76(\mathrm{~m}, 2 \mathrm{H}), 0.139(\mathrm{~s}, 3 \mathrm{H}), 0.136(\mathrm{~s}$, $3 \mathrm{H}) ;{ }^{13} \mathrm{C} \mathrm{NMR}\left(125 \mathrm{MHz}, \mathrm{CDCl}_{3}\right) \delta 143.04,143.02,129.0,128.4,125.2,86.5,83.9,60.5,53.7,47.2,46.9$, $36.4,36.2,34.3,32.5,32.0,31.8,31.4,27.7,25.9,24.1,23.9,-0.31,-0.36$; IR (thin film) $2951,2868,1597$, $1250 \mathrm{~cm}^{-1}$; HRMS (ESI/Na) $\mathrm{m} / z$ calcd for $\mathrm{C}_{33} \mathrm{H}_{50} \mathrm{O}_{2} \mathrm{SiNa}(\mathrm{M}+\mathrm{Na}) 529.3478$, found 529.3475. Anal. Calcd for $\mathrm{C}_{33} \mathrm{H}_{50} \mathrm{O}_{2} \mathrm{Si}: \mathrm{C}, 78.20 ; \mathrm{H}, 9.94$. Found: C, 77.49; H, 9.83. 

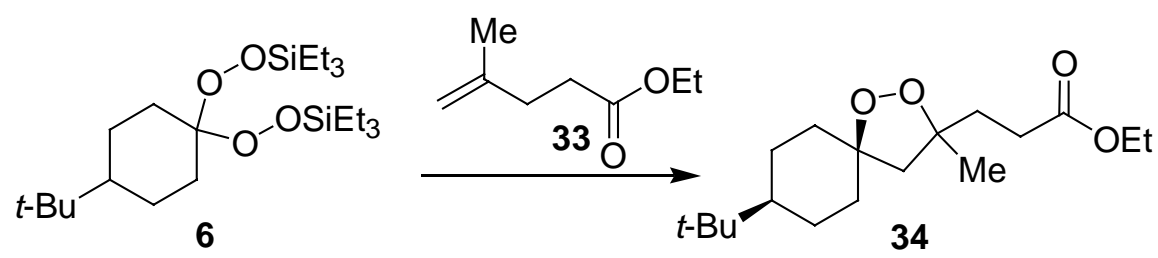

3-(8-tert-Butyl-3-methyl-1,2-dioxaspiro[4.5]dec-3-yl)-propionic acid ethyl ester (34). The standard annulation procedure was followed with silyl peroxyketal $6(0.0500 \mathrm{~g}, 0.116 \mathrm{mmol})$ and 4-methyl-pent-4-enoic acid ethyl ester $33(0.0493 \mathrm{~g}, 0.347 \mathrm{mmol})$ in $5 \mathrm{~mL}$ of $\mathrm{CH}_{2} \mathrm{Cl}_{2}$ and $\mathrm{SnCl}_{4}(0.23 \mathrm{~mL})$. Purification by flash chromatography (100:0 - 80:20 pentane/EtOAc) afforded 34 as a clear liquid $(0.0124 \mathrm{~g}, 34 \%)$ : ${ }^{1} \mathrm{H}$ NMR (500 $\left.\mathrm{MHz}, \mathrm{CDCl}_{3}\right) \delta 4.13(\mathrm{q}, J=7.2,2 \mathrm{H}), 2.40(\mathrm{~m}, 2 \mathrm{H}), 2.12(\mathrm{~d}, J=12.0,1 \mathrm{H}), 2.08(\mathrm{~m}, 2 \mathrm{H}), 2.05(\mathrm{~d}, J=12.0,1 \mathrm{H})$, $1.99(\mathrm{~m}, 1 \mathrm{H}), 1.83(\mathrm{ddd}, J=14.1,10.6,5.8,1 \mathrm{H}), 1.60(\mathrm{~m}, 2 \mathrm{H}), 1.46(\mathrm{~m}, J=13.6,4.0,1 \mathrm{H}), 1.34(\mathrm{~m}, 2 \mathrm{H}), 1.29$ $(\mathrm{s}, 3 \mathrm{H}), 1.25(\mathrm{t}, J=7.1,3 \mathrm{H}), 0.95(\mathrm{~m}, 1 \mathrm{H}), 0.86(\mathrm{~m}, 1 \mathrm{H}), 0.83(\mathrm{~s}, 9 \mathrm{H}) ;{ }^{13} \mathrm{C} \mathrm{NMR}\left(125 \mathrm{MHz}, \mathrm{CDCl}_{3}\right) \delta 173.8$, 84.7, 84.4, 60.7, 57.6, 47.3, 36.3, 36.0, 34.3, 32.6, 29.8, 27.7, 24.1, 23.9, 23.7, 14.4; IR (thin film) 2943, 2870, $1735,1443 \mathrm{~cm}^{-1}$; HRMS (ESI/Na) $\mathrm{m} / z$ calcd for $\mathrm{C}_{18} \mathrm{H}_{32} \mathrm{O}_{4} \mathrm{Na}(\mathrm{M}+\mathrm{Na}) 335.2198$, found 335.2201. Anal. Calcd for $\mathrm{C}_{18} \mathrm{H}_{32} \mathrm{O}_{4}$ : C, 69.19; H, 10.13 Found: C, 68.42; H, 10.45 .
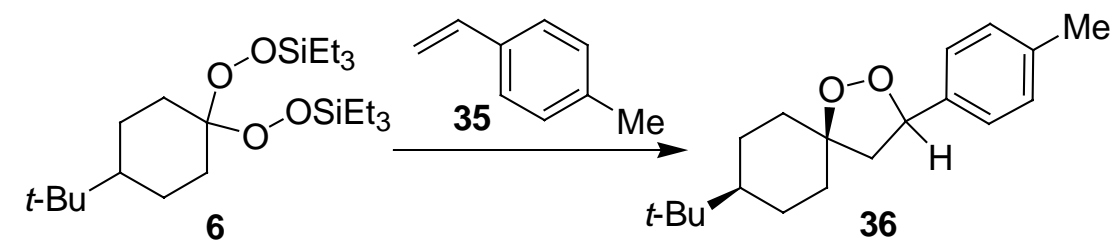

8-tert-Butyl-3-p-tolyl-1,2-dioxaspiro[4.5]decane (36). The standard annulation procedure was followed with silyl peroxyketal $6(0.050 \mathrm{~g}, 0.116 \mathrm{mmol})$ and $p$-methylstyrene $35(0.046 \mathrm{~g}, 0.348 \mathrm{mmol})$ in 5 $\mathrm{mL}$ of $\mathrm{CH}_{2} \mathrm{Cl}_{2}$ and $\mathrm{SnCl}_{4}(0.23 \mathrm{~mL})$. Purification by flash chromatography (100:0 - 80:20 hexanes/EtOAc) afforded 36 a clear liquid $(0.0094 \mathrm{~g}, 28 \%)$ : ${ }^{1} \mathrm{H}$ NMR $\left(500 \mathrm{MHz}, \mathrm{CDCl}_{3}\right) \delta 7.26(\mathrm{~d}, J=8.8,2 \mathrm{H}), 7.15(\mathrm{~d}, J=$ $7.9,2 \mathrm{H}), 5.22(\mathrm{t}, J=7.7,1 \mathrm{H}), 2.67(\mathrm{dd}, J=12.0,7.8,1 \mathrm{H}), 2.33(\mathrm{~s}, 3 \mathrm{H}), 2.31(\mathrm{dd}, \mathrm{J}=12.0,7.6,1 \mathrm{H}), 2.10(\mathrm{~m}$, $2 \mathrm{H}), 1.64(\mathrm{~m}, 2 \mathrm{H}), 1.50(\mathrm{~m}, 2 \mathrm{H}), 1.36(\mathrm{~m}, 1 \mathrm{H}), 0.97(\mathrm{~m}, 2 \mathrm{H}), 0.85(\mathrm{~s}, 9 \mathrm{H}) ;{ }^{13} \mathrm{C} \mathrm{NMR}\left(125 \mathrm{MHz} \mathrm{CDCl}_{3}\right) \delta$ 138.5, 136.1 , 129.5, 126.6, 84.9, 82.8, 54.8, 47.5, 36.4, 36.1, 32.6, 27.8, 24.2, 23.9, 21.4; IR (thin film) 2939, $1455,1043 \mathrm{~cm}^{-1}$; HRMS (ESI/Na) $m / z$ calcd for $\mathrm{C}_{19} \mathrm{H}_{28} \mathrm{O}_{2} \mathrm{Na}(\mathrm{M}+\mathrm{Na}) 311.1987$, found 311.1992. Anal. Calcd for $\mathrm{C}_{19} \mathrm{H}_{28} \mathrm{O}_{2}$ : C, 79.12; H, 9.78. Found: C, 76.39; H, 9.77.

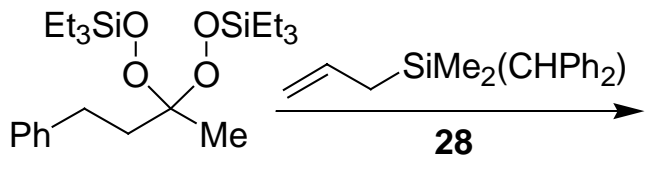

19

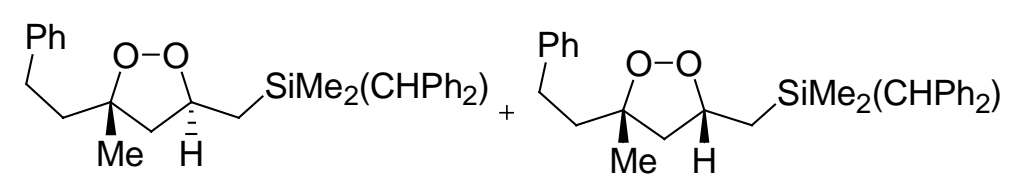

trans-38
$2: 1$

cis-38

Benzhydryldimethyl-(5-methyl-5-phenethyl[1.2]dioxolan-3-ylmethyl)-silane (38). To a $-78{ }^{\circ} \mathrm{C}$ solution of silyl peroxyketal $19(0.0500 \mathrm{~g}, 0.114 \mathrm{mmol})$ in $1 \mathrm{~mL}$ of $\mathrm{CH}_{2} \mathrm{Cl}_{2}$, was added benzhydryldimethylallylsilane $28(0.0305 \mathrm{~g}, 0.114 \mathrm{mmol})$, and $\mathrm{SnCl}_{4}(0.11 \mathrm{~mL})$. The reaction mixture was allowed to warm to $-5{ }^{\circ} \mathrm{C}$ over $2 \mathrm{~h}$ then added to $5 \mathrm{~mL}$ of ice-cold $\mathrm{H}_{2} \mathrm{O}$. The reaction mixture was extracted with $3 \times 20 \mathrm{~mL}$ of $\mathrm{CH}_{2} \mathrm{Cl}_{2}$. The combined organic layers were concentrated in vacuo. Purification by flash chromatography (80:20 hexanes $\left./ \mathrm{CH}_{2} \mathrm{Cl}_{2}\right)$ afforded trans-38 and cis-38 as a clear liquid $(0.0280$ g, 57\%, d.r. 2:1; major diastereomer isolated): IR (thin film) 3059, 2953, 1593, 1493, $1248 \mathrm{~cm}^{-1} ; \mathrm{HRMS}\left(\mathrm{CI} / \mathrm{NH}_{3}\right) \mathrm{m} / \mathrm{z}$ calcd for 
$\mathrm{C}_{28} \mathrm{H}_{38} \mathrm{O}_{2} \mathrm{NSi}\left(\mathrm{M}+\mathrm{NH}_{4}\right)$ 448.2672, found 448.2661. Anal. Calcd for $\mathrm{C}_{28} \mathrm{H}_{34} \mathrm{O}_{2} \mathrm{Si}: \mathrm{C}, 78.09 ; \mathrm{H}, 7.96$. Found: $\mathrm{C}$, 77.56; H, 8.00.

Major diastereomer (trans-38). ${ }^{1} \mathrm{H}$ NMR $\left(500 \mathrm{MHz}, \mathrm{CDCl}_{3}\right) \delta 7.27(\mathrm{~m}, 10 \mathrm{H}), 7.17(\mathrm{~m}, 3 \mathrm{H}), 7.15(\mathrm{~m}, 2 \mathrm{H})$, $4.21(\mathrm{~m}, 1 \mathrm{H}), 3.60(\mathrm{~s}, 1 \mathrm{H}), 2.64(\mathrm{~m}, 2 \mathrm{H}), 2.37(\mathrm{dd}, J=11.7,7.0,1 \mathrm{H}), 1.85(\mathrm{~m}, 2 \mathrm{H}), 1.73(\mathrm{dd}, J=11.7,7.9,1 \mathrm{H})$, $1.34(\mathrm{~s}, 3 \mathrm{H}), 1.12(\mathrm{dd}, J=14.2,6.2,1 \mathrm{H}), 0.86(\mathrm{dd}, J=14.2,8.4,1 \mathrm{H}), 0.11(\mathrm{~s}, 3 \mathrm{H}), 0.10(\mathrm{~s}, 3 \mathrm{H}) ;{ }^{13} \mathrm{C}$ NMR $(125$ $\left.\mathrm{MHz}, \mathrm{CDCl}_{3}\right) \delta 142.4,142.1,129.0,128.8,128.63,128.60,128.58,128.4,126.1,125.6,125.5,85.8,79.9,53.3$, $45.6,40.8,30.9,25.8,19.5,-2.45,-2.47$.

Minor diastereomer (cis-38). ${ }^{1} \mathrm{H}$ NMR $\left(500 \mathrm{MHz}, \mathrm{CDCl}_{3}\right) \delta 7.27(\mathrm{~m}, 10 \mathrm{H}), 7.17(\mathrm{~m}, 3 \mathrm{H}), 7.15(\mathrm{~m}, 2 \mathrm{H}), 4.28$ $(\mathrm{m}, 1 \mathrm{H}) 3.60(\mathrm{~s}, 1 \mathrm{H}), 2.64(\mathrm{~m}, 2 \mathrm{H}), 2.29(\mathrm{dd}, J=11.7,6.7,1 \mathrm{H}), 1.95(\mathrm{~m}, J=8.6,4.8,1 \mathrm{H}), 1.85(\mathrm{~m}, 2 \mathrm{H}), 1.34$ $(\mathrm{s}, 3 \mathrm{H}), 1.12(\mathrm{dd}, J=15.2,6.2,1 \mathrm{H}), 0.86(\mathrm{dd}, J=14.3,5.9,1 \mathrm{H}), 0.11(\mathrm{~s}, 3 \mathrm{H}), 0.10(\mathrm{~s}, 3 \mathrm{H}) ;{ }^{13} \mathrm{C}$ NMR $(125$ $\mathrm{MHz}, \mathrm{CDCl}_{3}$, distinctive peaks) $\delta$ 85.6, 79.6, 53.7, 45.4, 42.4, 30.96, 23.6, 19.2.

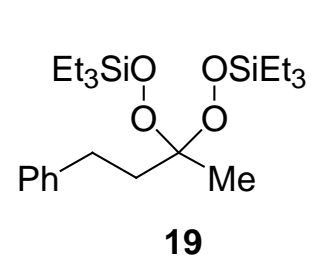

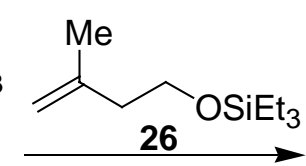

19

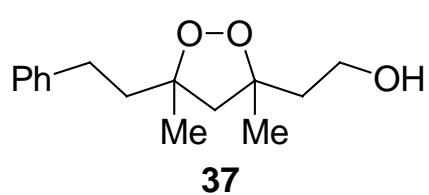

37

2-(3,5-Dimethyl-5-phenethyl[1.2] dioxolan-3-yl)-ethanol (37). The standard annulation procedure was followed with silyl peroxyketal $19(0.0500 \mathrm{~g}, 0.114 \mathrm{mmol})$ and triethyl-(3-methyl-but-3-enyloxy)-silane 26 $(0.0685 \mathrm{~g}, 0.342 \mathrm{mmol})$ in $5 \mathrm{~mL}$ of $\mathrm{CH}_{2} \mathrm{Cl}_{2}$ and $\mathrm{SnCl}_{4}(0.23 \mathrm{~mL})$. Purification by flash chromatography (100:0 $-50: 50$ pentane/pentane) afforded 37 as a clear liquid (0.0192 g, 67\%, d.r. 1:1): ${ }^{1} \mathrm{H}$ NMR $\left(500 \mathrm{MHz}, \mathrm{CDCl}_{3}\right) \delta$ $7.27(\mathrm{~m}, 4 \mathrm{H}), 7.18(\mathrm{~m}, 6 \mathrm{H}), 3.77(\mathrm{~m}, 4 \mathrm{H}), 2.67(\mathrm{~m}, 4 \mathrm{H}), 2.32(\mathrm{~m}, 4 \mathrm{H}), 2.16(\mathrm{~m}, 2 \mathrm{H}), 2.00(\mathrm{~m}, 4 \mathrm{H}), 1.83(\mathrm{~m}$, $4 \mathrm{H}), 1.38(\mathrm{~m}, 12 \mathrm{H}) ;{ }^{13} \mathrm{C} \mathrm{NMR}\left(125 \mathrm{MHz}, \mathrm{CDCl}_{3}\right) \delta 142.0,141.9,128.6,128.41,128.39,126.07,126.03,86.14$, $86.12,86.0,59.33,59.31,56.9,56.8,41.58,41.56,41.50,41.16,31.13,30.99,24.49,24.34,24.26,23.6$; IR (thin film) 3373, 3028, 2934, $1456 \mathrm{~cm}^{-1}$; HRMS (ESI/Na) $\mathrm{m} / z$ calcd for $\mathrm{C}_{15} \mathrm{H}_{22} \mathrm{O}_{3} \mathrm{Na}(\mathrm{M}+\mathrm{Na}) 273.1467$, found 273.1470. Anal. Calcd for $\mathrm{C}_{15} \mathrm{H}_{22} \mathrm{O}_{3}: \mathrm{C}, 71.97 ; \mathrm{H}, 8.86$. Found: C, 71.70; H, 9.01.

\section{Oxidation of the Silane Moiety ${ }^{7}$}

General Procedure for the oxidation of the silane moiety. To a solution of 1,2-dioxolane (1.0 equiv) in THF $(1.0 \mathrm{M})$ was added $n$-Bu $\mathrm{Bu}_{4} \mathrm{NF}$ (1.6 equiv, $1.0 \mathrm{M}$ solution in THF) dropwise by syringe at $0{ }^{\circ} \mathrm{C}$. The ice bath was removed and the reaction mixture was allowed to warm to ambient temperature. After $30 \mathrm{~min}, \mathrm{MeOH}(53$ equiv) was added to the reaction mixture followed by $\mathrm{KHCO}_{3}$ (1.5 equiv) and $\mathrm{H}_{2} \mathrm{O}_{2}$ (10 equiv, $30 \%$ wt. $\mathrm{H}_{2} \mathrm{O}$ ). After $12 \mathrm{~h}-24 \mathrm{~h}$, the reaction mixture was diluted with $\mathrm{CH}_{2} \mathrm{Cl}_{2} / \mathrm{H}_{2} \mathrm{O}(1: 1)$, the layers were separated, and the aqueous layer was extracted with $\mathrm{CH}_{2} \mathrm{Cl}_{2}$. The combined organic layers were dried $\left(\mathrm{MgSO}_{4}\right)$, filtered, and concentrated in vacuo. Purification by flash chromatography was performed using Davisil ${ }^{\circledR}$ silica gel.
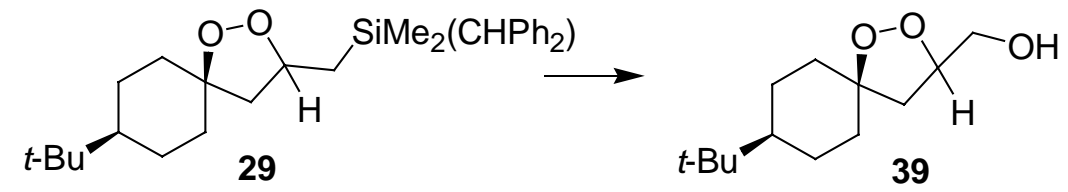
(8-tert-Butyl-1,2-dioxaspiro[4.5]dec-3-yl)-methanol (39). The standard oxidation procedure was followed with $29(0.250 \mathrm{~g}, 0.570 \mathrm{mmol})$ in $5 \mathrm{~mL}$ of THF, $n$-Bu ${ }_{4} \mathrm{NF}(0.9 .2 \mathrm{~mL}), \mathrm{MeOH}(1.30 \mathrm{~mL}), \mathrm{KHCO}_{3}$ $(0.0860 \mathrm{~g}, 0.860 \mathrm{mmol})$ and $\mathrm{H}_{2} \mathrm{O}_{2}(0.72 \mathrm{~mL}, 5.7 \mathrm{mmol})$. Purification by flash chromatography (80:20 hexanes/EtOAc) afforded 39 as a white solid (0.0683 g, 52\%): mp 58.0-59.0 ${ }^{\circ} \mathrm{C} ;{ }^{1} \mathrm{H} \mathrm{NMR}\left(500 \mathrm{MHz}, \mathrm{CDCl}_{3}\right) \delta$ 4.40 (dddd, $J=8.1,7.3,4.7,3.3,1 \mathrm{H}), 3.60(\mathrm{~m}, 2 \mathrm{H}), 2.35(\mathrm{dd}, J=12.0,8.2,1 \mathrm{H}), 2.09(\mathrm{~m}, J=7.1,2.9,1 \mathrm{H}), 2.01$ $(\mathrm{dd}, J=12,4.8,1 \mathrm{H}), 1.89(\mathrm{~m}, 1 \mathrm{H}), 1.62(\mathrm{~m}, 3 \mathrm{H}), 1.33(\mathrm{~m}, 2 \mathrm{H}), 1.19(\mathrm{~m}, 2 \mathrm{H}), 0.98(\mathrm{~m}, 1 \mathrm{H}), 0.84(\mathrm{~s}, 9 \mathrm{H})) ;{ }^{13} \mathrm{C}$ NMR $\left(125 \mathrm{MHz}, \mathrm{CDCl}_{3}\right) \delta 84.0,81.0,63.8,47.4,47.1,35.5,35.0,32.4,27.5,24.3,23.4$; IR (thin film) 3316, 2938, 2862, 1451, $1039 \mathrm{~cm}^{-1}$; HRMS $\left(\mathrm{CI} / \mathrm{NH}_{3}\right) \mathrm{m} / \mathrm{z}$ calcd $\mathrm{C}_{13} \mathrm{H}_{24} \mathrm{O}_{3}(\mathrm{M}) 228.1725$ found 228.1720. Anal. Calcd for $\mathrm{C}_{13} \mathrm{H}_{24} \mathrm{O}_{3}$ : C, 68.38; H, 10.59. Found: C, 68.60; H, 10.61.

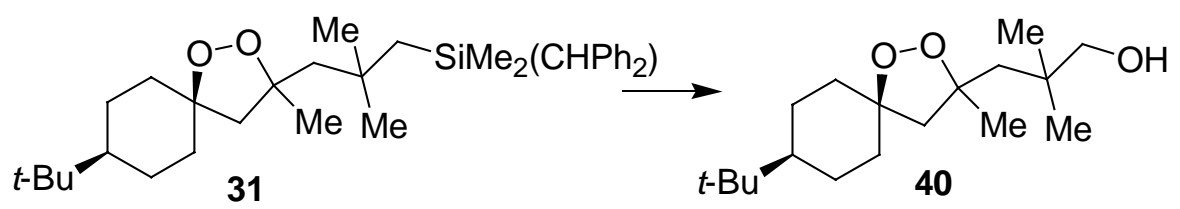

3-(8-tert-Butyl-3-methyl-1,2-dioxaspiro[4.5]dec-3-yl)-2,2-dimethylpropan-1-ol (40). The standard oxidation procedure was followed with $31(0.0650 \mathrm{~g}, 0.128 \mathrm{mmol})$ in $1.4 \mathrm{~mL}$ of $\mathrm{THF}, n-\mathrm{Bu}_{4} \mathrm{NF}(0.46 \mathrm{~mL})$, $\mathrm{MeOH}(0.280 \mathrm{~mL}), \mathrm{KHCO}_{3}(0.0226 \mathrm{mg}, 0.216 \mathrm{mmol})$ and $\mathrm{H}_{2} \mathrm{O}_{2}(0.083 \mathrm{~mL}, 1.4 \mathrm{mmol})$. Purification by flash chromatography (100:0 - 90:10 pentane/EtOAc) afforded 40 as white solid (0.0200 g, 52\%): $\mathrm{mp} 79.5-80.5^{\circ} \mathrm{C}$; ${ }^{1} \mathrm{H}$ NMR $\left(500 \mathrm{MHz}, \mathrm{CDCl}_{3}\right) \delta 3.57(\mathrm{dd}, J=11.3,5.5,1 \mathrm{H}), 3.21(\mathrm{dd}, J=11.1,8.9,1 \mathrm{H}), 2.51(\mathrm{dd}, J=8.5,5.7$, $1 \mathrm{H}), 2.10(\mathrm{~m}, 3 \mathrm{H}), 1.97(\mathrm{~m}, 2 \mathrm{H}), 1.59(\mathrm{~d}, J=12.4,2 \mathrm{H}), 1.45(\mathrm{~m}, 1 \mathrm{H}), 1.43(\mathrm{~s}, 3 \mathrm{H}), 1.29(\mathrm{~m}, 4 \mathrm{H}), 0.97(\mathrm{~s}, 3 \mathrm{H})$, $0.95(\mathrm{~s}, 3 \mathrm{H}), 0.93(\mathrm{~m}, 1 \mathrm{H}), 0.82(\mathrm{~s}, 9 \mathrm{H}) ;{ }^{13} \mathrm{C} \mathrm{NMR}\left(125 \mathrm{MHz}, \mathrm{CDCl}_{3}\right) \delta 86.8,84.5,71.3,61.8,47.4,47.2,36.6$, $36.3,36.2,32.6,28.8,27.7,25.4,25.3,24.2,23.9$; IR (thin film) 3340, 2967, 2866, 1460, $1047 \mathrm{~cm}^{-1}$; HRMS (ESI/Na) $m / z$ calcd $\mathrm{C}_{18} \mathrm{H}_{34} \mathrm{O}_{3} \mathrm{Na}(\mathrm{M}+\mathrm{Na}) 321.2406$, found 321.2412. Anal. Calcd for $\mathrm{C}_{18} \mathrm{H}_{34} \mathrm{O}_{3}: \mathrm{C}, 72.44 ; \mathrm{H}$, 11.48. Found: $\mathrm{C}, 72.71 ; \mathrm{H}, 11.77$.

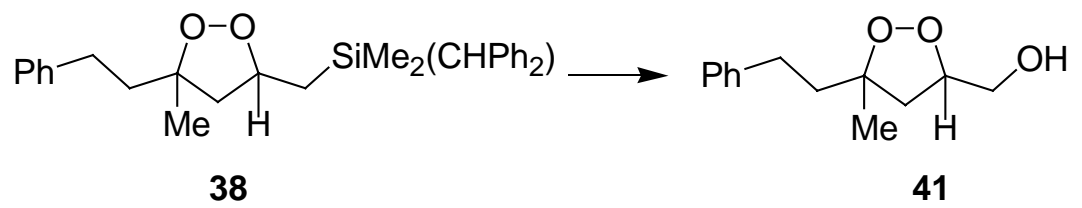

(5-Methyl-5-phenethyl[1.2]dioxolan-3-yl)-methanol (41). The standard oxidation procedure was followed with $38(0.280 \mathrm{~g}, 0.650 \mathrm{mmol})$ in $6 \mathrm{~mL}$ of THF, $n$-Bu $\mathrm{H}_{4} \mathrm{NF}(1.0 \mathrm{~mL}), \mathrm{MeOH}(1.20 \mathrm{~mL}), \mathrm{KHCO}_{3}$ (0.0976 g, $0.975 \mathrm{mmol})$ and $\mathrm{H}_{2} \mathrm{O}_{2}(0.38 \mathrm{~mL}, 6.5 \mathrm{mmol})$. Purification by flash chromatography $(100: 0-90: 10$ pentane/EtOAc) afforded 41 as a clear liquid $\left(0.0860 \mathrm{~g}, 60 \%\right.$, d.r. 2:1; mixture of diastereomers): ${ }^{1} \mathrm{H}$ NMR (500 $\left.\mathrm{MHz}, \mathrm{CDCl}_{3}\right) \delta 7.27(\mathrm{~m}, 4 \mathrm{H}), 7.19(\mathrm{~m}, 6 \mathrm{H}), 4.46(\mathrm{~m}, 1 \mathrm{H}), 4.41(\mathrm{~m}, 1 \mathrm{H}), 3.65(\mathrm{~m}, 4 \mathrm{H}), 2.69(\mathrm{~m}, 4 \mathrm{H}), 2.52(\mathrm{dd}, J$ $=11.7,8.3,1 \mathrm{H}), 2.41(\mathrm{dd}, \mathrm{J}=12.0,8.1,1 \mathrm{H}), 2.31(\mathrm{~m}, 2 \mathrm{H}), 2.16(\mathrm{dd}, \mathrm{J}=12.0,5.4,1 \mathrm{H}), 2.10(\mathrm{dd}, J=12.2,4.8$, $1 \mathrm{H}), 1.93(\mathrm{~m}, 2 \mathrm{H}), 1.83(\mathrm{~m}, 2 \mathrm{H}) 1.40(\mathrm{~s}, 3 \mathrm{H}), 1.37(\mathrm{~s}, 3 \mathrm{H}) ;{ }^{13} \mathrm{C} \mathrm{NMR}\left(125 \mathrm{MHz}, \mathrm{CDCl}_{3}\right) \delta 142.0,141.7,128.6$, 128.5, 128.4, 128.3, 126.1, 126.0, 85.4, 85.2, 81.9, 81.8, 63.7, 63.6, 47.0, 46.6, 41.1, 40.1, 31.2, 30.7, 24.2, 22.4; IR (thin film) 3406, 3017, 2946, 1455, $\mathrm{cm}^{-1}$; HRMS (EI) $\mathrm{m} / \mathrm{z}$ calcd $\mathrm{C}_{13} \mathrm{H}_{18} \mathrm{O}_{3}$ (M) 222.1256, found 222.1253. Anal. Calcd for $\mathrm{C}_{13} \mathrm{H}_{18} \mathrm{O}_{3}$ : C, 70.24; H, 8.16. Found: C, 70.01; H, 8.25. 


\section{Oxidation of Hydroxyl Groups}<smiles>CC(C)(C)[C@H]1CC[C@]2(CC1)C[C@](C)(CCO)OO2</smiles>

(8-tert-Butyl-3-methyl-1,2-dioxaspiro[4.5]dec-3-yl)-acetic acid (42a). To a solution of 27 (0.0370 g, $0.144 \mathrm{mmol})$ in $1 \mathrm{~mL}$ of acetonitrile was added $\mathrm{H}_{5} \mathrm{IO}_{6}(0.0790 \mathrm{~g}, 0.346 \mathrm{mmol}), \mathrm{RuCl}_{3} \cdot \mathrm{H}_{2} \mathrm{O}(0.001 \mathrm{~g}, 0.005$ mmol), $1 \mathrm{~mL}$ of $\mathrm{CCl}_{4}$ and $1 \mathrm{~mL}$ of $\mathrm{H}_{2} \mathrm{O}{ }^{10}$ The reaction mixture was stirred for $1.5 \mathrm{~h}$ at ambient temperature then diluted with $10 \mathrm{~mL}$ of $\mathrm{CH}_{2} \mathrm{Cl}_{2}$ and $10 \mathrm{~mL}$ of $\mathrm{H}_{2} \mathrm{O}$. The layers were separated, and the aqueous layer was extracted with $3 \times 10 \mathrm{~mL}$ of $\mathrm{CH}_{2} \mathrm{Cl}_{2}$. The combined organic layers were dried $\left(\mathrm{Na}_{2} \mathrm{SO}_{4}\right)$, filtered, and concentrated in vacuo. Purification by celite-filtration afforded 42a as a white solid $(0.0339 \mathrm{~g}, 87 \%): \mathrm{mp} 113-$ $114{ }^{\circ} \mathrm{C} ;{ }^{1} \mathrm{H}$ NMR $\left(500 \mathrm{MHz}, \mathrm{CDCl}_{3}\right) \delta 12.1-9.6(\mathrm{bs}, 1 \mathrm{H}), 2.78(\mathrm{~d}, J=14.9,1 \mathrm{H}), 2.72(\mathrm{~d}, J=14.8,1 \mathrm{H}), 2.41(\mathrm{~d}$, $J=12.4,1 \mathrm{H}), 2.12(\mathrm{~m}, 2 \mathrm{H}), 1.94(\mathrm{~m}, 1 \mathrm{H}), 1.61(\mathrm{~m}, 2 \mathrm{H}), 1.53(\mathrm{~m}, J=13.7,4.1,1 \mathrm{H}), 1.46(\mathrm{~s}, 3 \mathrm{H}), 1.34(\mathrm{~m}, 2 \mathrm{H})$, $1.24(\mathrm{~m}, 1 \mathrm{H}), 0.96(\mathrm{~m}, 1 \mathrm{H}), 0.84(\mathrm{~s}, 9 \mathrm{H}) ;{ }^{13} \mathrm{C} \mathrm{NMR}\left(125 \mathrm{MHz}, \mathrm{CDCl}_{3}\right) \delta$ 176.42, 85.0, 83.2, 56.6, 47.0, 44.0, 36.0, 35.7, 32.4, 27.5, 24.0, 23.8, 23.5; IR (thin film) 2943, 2870, $1738 \mathrm{~cm}^{-1}$; HRMS (ESI/Na) $\mathrm{m} / \mathrm{z}$ calcd $\mathrm{C}_{15} \mathrm{H}_{26} \mathrm{O}_{4} \mathrm{Na}(\mathrm{M}+\mathrm{Na}) 293.1729$, found 293.1721.

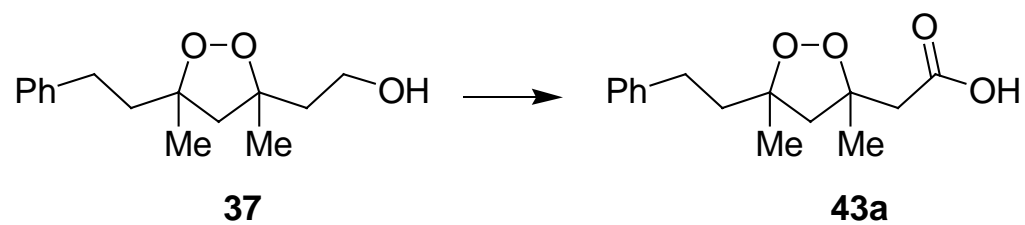

(3,5-Dimethyl-5-phenethyl[1.2]dioxolan-3-yl)-acetic acid (43a). To a solution of 37 (0.0500 g, 0.199 mmol) in $1.3 \mathrm{~mL}$ of acetonitrile was added $\mathrm{H}_{5} \mathrm{IO}_{6}(0.109 \mathrm{~g}, 0.478 \mathrm{mmol}), \mathrm{RuCl}_{3} \cdot \mathrm{H}_{2} \mathrm{O}(0.001 \mathrm{~g}, 0.007 \mathrm{mmol})$, $1.3 \mathrm{~mL}$ of $\mathrm{CCl}_{4}$ and $2 \mathrm{~mL}$ of $\mathrm{H}_{2} \mathrm{O} .{ }^{10}$ The reaction mixture was stirred for $1.5 \mathrm{~h}$ at ambient temperature then diluted with $10 \mathrm{~mL}$ of $\mathrm{CH}_{2} \mathrm{Cl}_{2}$ and $10 \mathrm{~mL}$ of $\mathrm{H}_{2} \mathrm{O}$. The layers were separated, and the aqueous layer was extracted with $3 \times 10 \mathrm{~mL}$ of $\mathrm{CH}_{2} \mathrm{Cl}_{2}$. The combined organic layers were dried $\left(\mathrm{Na}_{2} \mathrm{SO}_{4}\right)$, filtered, and concentrated in vacuo. Purification by celite-filtration afforded 43a as a clear oil $(0.0380 \mathrm{~g}, 72 \%$, d.r. $1: 1$; mixture of diastereomers): ${ }^{1} \mathrm{H}$ NMR $\left(500 \mathrm{MHz}, \mathrm{CDCl}_{3}\right) \delta 7.28(\mathrm{~m}, 4 \mathrm{H}), 7.19(\mathrm{~m}, 6 \mathrm{H}), 2.85-2.52(\mathrm{~m}, 8 \mathrm{H}), 2.42-$ $2.04(\mathrm{~m}, 4 \mathrm{H}), 2.00-1.80(\mathrm{~m}, 4 \mathrm{H}), 1.51(\mathrm{~s}, 3 \mathrm{H}), 1.47(\mathrm{~s}, 3 \mathrm{H}), 1.43(\mathrm{~s}, 3 \mathrm{H}), 1.38(\mathrm{~s}, 3 \mathrm{H}) ;{ }^{13} \mathrm{C} \mathrm{NMR}(125 \mathrm{MHz}$, $\left.\mathrm{CDCl}_{3}\right) \delta 175.4,141.9,141.6,128.49,128.46,128.31,128.26,126.0,125.9,86.3,86.2,83.9,83.8,55.9,55.5$, 43.92, 43.89, 41.7, 40.7, 31.0, 30.7, 24.7, 23.9, 23.6, 23.0; IR (thin film) 3028, 2936, 1718, $1454 \mathrm{~cm}^{-1}$; HRMS (ESI/Na) $m / z$ calcd $\mathrm{C}_{15} \mathrm{H}_{20} \mathrm{O}_{4} \mathrm{Na}(\mathrm{M}+\mathrm{Na}) 287.1259$, found 287.1253.<smiles>CC1(CC(=O)O)CC2(CCC(C(C)(C)C)CC2)OO1</smiles><smiles>COC(=O)CC1(C)CC2(CCC(C(C)(C)C)CC2)OO1</smiles>

(8-tert-Butyl-3-methyl-1,2-dioxaspiro[4.5]dec-3-yl)-acetic acid methyl ester (42). To a solution of 42a $(0.0270 \mathrm{~g}, 0.0999 \mathrm{mmol})$ in $1 \mathrm{~mL}$ of hexanes and $1 \mathrm{~mL}$ of $\mathrm{CH}_{3} \mathrm{OH}$ was added trimethylsilyl diazomethane $(0.0950 \mathrm{~mL}, 0.190 \mathrm{mmol})$ at ambient temperature. ${ }^{11}$ The reaction mixture was stirred for $20 \mathrm{~min}$ and acetic acid 
was added dropwise until the yellow color disappeared. The solvent was removed in vacuo to afford $\mathbf{4 2}$ as a clear liquid (0.0270 g, 95\%): ${ }^{1} \mathrm{H}$ NMR $\left(500 \mathrm{MHz}, \mathrm{CDCl}_{3}\right) \delta 3.67(\mathrm{~s}, 3 \mathrm{H}), 2.72(\mathrm{~d}, J=14.6,1 \mathrm{H}), 2.66(\mathrm{~d}, J=$ $14.6,1 \mathrm{H}), 2.42(\mathrm{~d}, J=15.5,1 \mathrm{H}), 2.10(\mathrm{~m}, 2 \mathrm{H}), 1.95(\mathrm{~m}, 1 \mathrm{H}), 1.60(\mathrm{~m}, 2 \mathrm{H}), 1.53(\mathrm{~m}, J=13.5,4.2,1 \mathrm{H}), 1.43(\mathrm{~s}$, $3 \mathrm{H}), 1.34(\mathrm{~m}, 2 \mathrm{H}), 1.24(\mathrm{~m}, 1 \mathrm{H}), 0.96(\mathrm{~m}, 1 \mathrm{H}), 0.83(\mathrm{~s}, 9 \mathrm{H}) ;{ }^{13} \mathrm{C} \mathrm{NMR}\left(125 \mathrm{MHz}, \mathrm{CDCl}_{3}\right) \delta 171.3,85.1,83.5$, 56.7, 51.9, 47.2, 44.3, 36.2, 35.9, 32.6, 27.7, 24.2, 24.1, 23.7; IR (thin film) 2941, 2867, 2736, 2638, $1694 \mathrm{~cm}^{-1}$; HRMS (ESI/Na) $m / z$ calcd $\mathrm{C}_{16} \mathrm{H}_{28} \mathrm{O}_{4} \mathrm{Na}(\mathrm{M}+\mathrm{Na})$ 307.1885, found 307.1890. Anal. Calcd for $\mathrm{C}_{16} \mathrm{H}_{28} \mathrm{O}_{4}: \mathrm{C}_{\text {, }}$ 67.57; H, 9.92. Found: C, 67.57; H, 9.99.

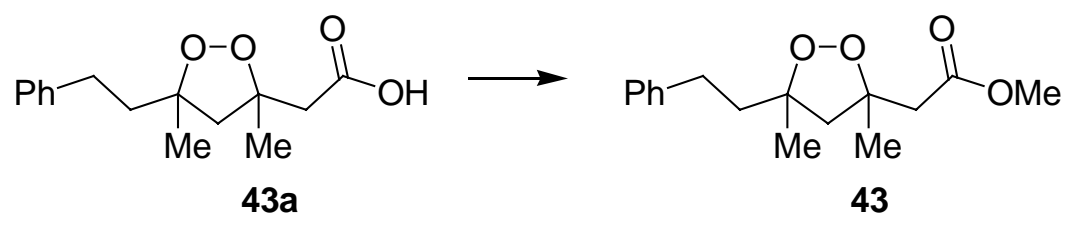

(3,5-Dimethyl-5-phenethyl[1.2]dioxolan-3-yl)-acetic acid methyl ester (43). To a solution of 43a $(0.0160 \mathrm{~g}, 0.0605 \mathrm{mmol})$ in $0.5 \mathrm{~mL}$ of hexanes and $0.1 \mathrm{~mL}$ of $\mathrm{CH}_{3} \mathrm{OH}$ was added trimethylsilyl diazomethane $(0.0575 \mathrm{~mL}, 0.115 \mathrm{mmol})$ at ambient temperature. ${ }^{11}$ The reaction mixture was stirred for $15 \mathrm{~min}$ and acetic acid was added dropwise until the yellow color disappeared. Solvent was removed in vacuo to afford $\mathbf{4 3}$ as a clear liquid $\left(0.0142 \mathrm{~g}, 85 \%\right.$, d.r. $1: 1$; mixture of diastereomers): ${ }^{1} \mathrm{H} \mathrm{NMR}\left(500 \mathrm{MHz}, \mathrm{CDCl}_{3}\right) \delta 7.28(\mathrm{~m}, 4 \mathrm{H}), 7.19(\mathrm{~m}$, $6 \mathrm{H}), 3.70(\mathrm{~s}, 3 \mathrm{H}), 3.68(\mathrm{~s}, 3 \mathrm{H}), 2.81-2.61(\mathrm{~m}, 9 \mathrm{H}), 2.55(\mathrm{~d}, J=12.5,1 \mathrm{H}), 2.27(\mathrm{~d}, J=12.5,1 \mathrm{H}), 2.18(\mathrm{~d}, J=$ $12.5,1 \mathrm{H}), 2.06(\mathrm{~m}, J=12.6,4.8,1 \mathrm{H}), 1.96(\mathrm{~m}, J=12.0,5.4,1 \mathrm{H}), 1.86(\mathrm{~m}, 2 \mathrm{H}), 1.48(\mathrm{~s}, 3 \mathrm{H}), 1.44(\mathrm{~s}, 3 \mathrm{H}), 1.42$ $(\mathrm{s}, 3 \mathrm{H}), 1.37(\mathrm{~s}, 3 \mathrm{H}) ;{ }^{13} \mathrm{C}$ NMR $\left(125 \mathrm{MHz}, \mathrm{CDCl}_{3}\right) \delta 171.1,171.0,142.0,141.8,128.46,128.45,128.31$, 128.27, 126.0, 125.9, 86.2, 86.1, 84.1, 84.0, 55.7, 55.3, 51.8, 51.7, 44.1, 44.0, 41.7, 40.7, 31.0, 30.1, 24.6, 24.2, 23.9, 23.1; IR (thin film) 3028, 2953, 1735, $1455 \mathrm{~cm}^{-1}$; HRMS (ESI/Na) $m / z$ calcd $\mathrm{C}_{16} \mathrm{H}_{22} \mathrm{O}_{4} \mathrm{Na}(\mathrm{M}+\mathrm{Na})$ 301.1416, found 301.1416. Anal. Calcd for $\mathrm{C}_{16} \mathrm{H}_{22} \mathrm{O}_{4}$ : C, 69.04; H, 7.97. Found: C, 69.16; H, 8.01.

\section{Stereochemical Proofs of Annulation Products}

A. The Stereochemistry of $\mathbf{2 5}$ was determined by analysis of nOe data:

Relevant DPFGSE-nOe data (mixing time $5.0 \mathrm{~s}$ ): (the peaks in the NMR spectrum were assigned using ${ }^{1} \mathrm{H} /{ }^{1} \mathrm{H}$ COSY, ${ }^{1} \mathrm{H} /{ }^{13} \mathrm{C}$ HMQC, ${ }^{1} \mathrm{H}$ NMR chemical shifts, and ${ }^{1} \mathrm{H}$ NMR coupling constants)
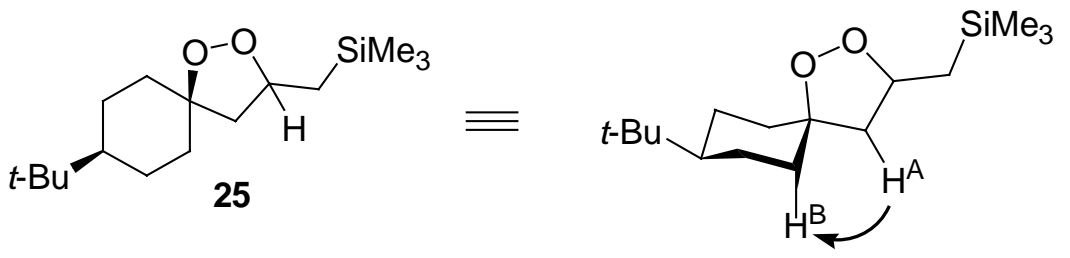

(8-tert-Butyl-1,2-dioxaspiro[4.5]dec-3-ylmethyl)trimethylsilane (25)

nOe experiments were performed for $\mathbf{2 5}$ using a single diastereomer $\mathbf{H}^{\mathrm{A}}$ irradiated: $\mathrm{H}^{\mathrm{B}}(10.8 \%)$

Note: nOe between $\mathrm{H}^{\mathrm{A}}$ and $\mathrm{H}^{\mathrm{B}}$ suggests a 1,4-cis configuration. Coupling constants suggest $\mathrm{H}^{\mathrm{B}}$ is in an axial position. 
B. The Stereochemistry of trans-38 was determined by analysis of nOe data:

Relevant DPFGSE-nOe data (mixing time 5.0 s): (the peaks in the NMR spectrum were assigned using ${ }^{1} \mathrm{H} /{ }^{1} \mathrm{H}$ COSY, ${ }^{1} \mathrm{H} /{ }^{13} \mathrm{C} \mathrm{HMQC}$, and ${ }^{1} \mathrm{H}$ NMR chemical shifts)

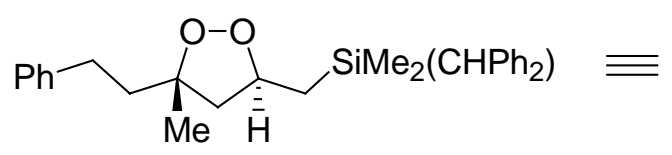

trans-38

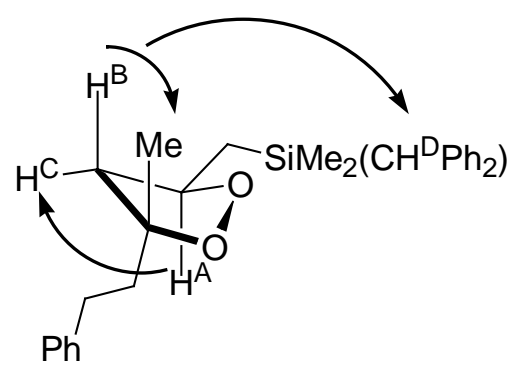

Benzhydryl-dimethyl-(5-methyl-5-phenethyl[1.2]dioxolan-3-ylmethyl)silane (trans-38) nOe experiments were performed for trans-38 using a single diastereomer $\mathbf{H}^{\mathbf{A}}$ irradiated: $\mathrm{H}^{\mathrm{C}}(4.3 \%)$ $\mathbf{H}^{\mathbf{B}}$ irradiated: $\mathrm{Me}(2.3 \%), \mathrm{H}^{\mathrm{D}}(1.7 \%)$

Note: nOe between $\mathrm{H}^{\mathrm{B}}$ and $\mathrm{H}^{\mathrm{D}}$ and absence of nOe between $\mathrm{H}^{\mathrm{A}}$ and $\mathrm{H}^{\mathrm{B}}$ suggests a 1,3-trans configuration.

\section{X-Ray Crystallographic Data}<smiles>CC(C)(CO)CC1(C)CC2(CCC(C(C)(C)C)CC2)OO1</smiles>

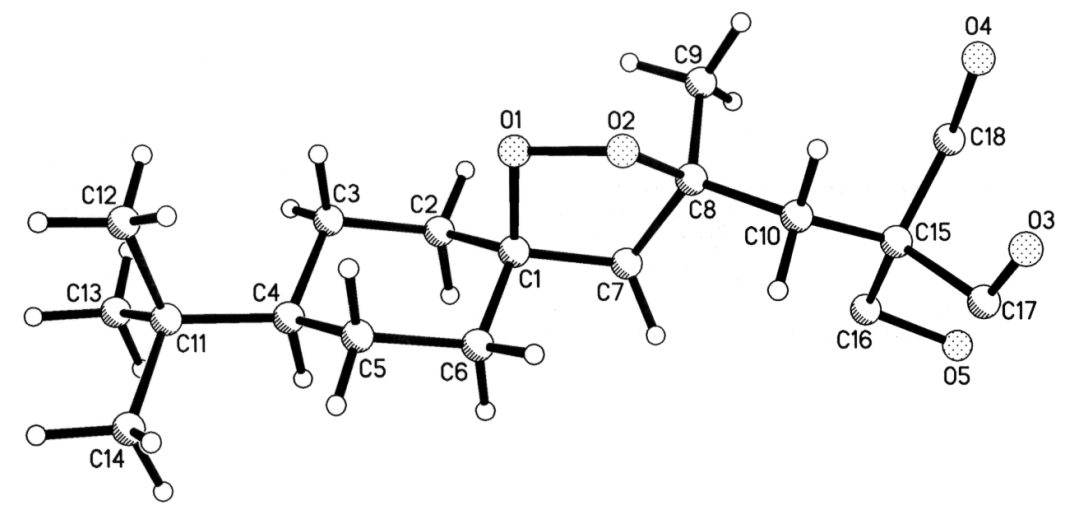

X-ray Data Collection, Structure Solution and Refinement for 3-(8-tert-Butyl-3-methyl-1,2dioxaspiro[4.5]dec-3-yl)-2,2-dimethylpropan-1-ol (40): 
A colorless crystal of approximate dimensions $0.13 \times 0.19 \times 0.27 \mathrm{~mm}$ was mounted on a glass fiber and transferred to a Bruker CCD platform diffractometer. The SMART ${ }^{1}$ program package was used to determine the unit-cell parameters and for data collection $(30 \mathrm{sec} /$ frame scan time for a sphere of diffraction data). The raw frame data was processed using $\mathrm{SAINT}^{2}$ and $\mathrm{SADABS}^{3}$ to yield the reflection data file. Subsequent calculations were carried out using the SHELXTL ${ }^{4}$ program. The diffraction symmetry was $2 / m$ and the systematic absences were consistent with the centrosymmetric monoclinic space group $P 2_{1} / c$ which was later determined to be correct.

The structure was solved by direct methods and refined on $\mathrm{F}^{2}$ by full-matrix least-squares techniques. The analytical scattering factors ${ }^{5}$ for neutral atoms were used throughout the analysis. Hydrogen atoms were either located from a difference-Fourier map and refined ( $\mathrm{x}, \mathrm{y}, \mathrm{z}$ and $\left.\mathrm{U}_{\mathrm{iso}}\right)$ or were included using a riding model. The $\mathrm{OH}$ was disordered over three positions and included with site-occupancy $=1 / 3$ at each position. Hydrogen atoms associated with $\mathrm{C} 16, \mathrm{C} 17$ and $\mathrm{C} 18$ were also included with partial site-occupancy consistent with the disordered model.

Final least-squares analysis yielded wR2 $=0.1891$ and Goof $=1.049$ for 337 variables refined against 3031 data $(0.85 \AA)$. As a comparison for refinement on $\mathrm{F}, \mathrm{R} 1=0.0666$ for those 1912 data with $\mathrm{I}>2.0 \sigma(\mathrm{I})$.

References.

1. SMART Software Users Guide, Version 5.1, Bruker Analytical X-Ray Systems, Inc.; Madison, WI 1999.

2. SAINT Software Users Guide, Version 6.0, Bruker Analytical X-Ray Systems, Inc.; Madison, WI 1999.

3. Sheldrick, G. M. SADABS, Version 2.10, Bruker Analytical X-Ray Systems, Inc.; Madison, WI 2002.

4. Sheldrick, G. M. SHELXTL Version 6.12, Bruker Analytical X-Ray Systems, Inc.; Madison, WI 2001.

5. International Tables for X-Ray Crystallography 1992, Vol. C., Dordrecht: Kluwer Academic Publishers.

Definitions:

$\mathrm{wR} 2=\left[\Sigma\left[\mathrm{w}\left(\mathrm{F}_{\mathrm{o}}^{2}-\mathrm{F}_{\mathrm{c}}^{2}\right)^{2}\right] / \Sigma\left[\mathrm{w}\left(\mathrm{F}_{\mathrm{o}}^{2}\right)^{2}\right]\right]^{1 / 2}$

$\mathrm{R} 1=\Sigma\left\|\mathrm{F}_{\mathrm{o}}|-| \mathrm{F}_{\mathrm{c}}\right\| / \Sigma\left|\mathrm{F}_{\mathrm{o}}\right|$

Goof $=\mathrm{S}=\left[\Sigma\left[\mathrm{w}\left(\mathrm{F}_{\mathrm{o}}^{2}-\mathrm{F}_{\mathrm{c}}{ }^{2}\right)^{2}\right] /(\mathrm{n}-\mathrm{p})\right]^{1 / 2}$ where $\mathrm{n}$ is the number of reflections and $\mathrm{p}$ is the total number of parameters refined.

The thermal ellipsoid plot is shown at the _\% probability level. 
Table 1. Crystal data and structure refinement for $\mathbf{4 0 .}$

Identification code

Empirical formula

Formula weight

Temperature

Wavelength

Crystal system

Space group

Unit cell dimensions

Volume

$\mathrm{Z}$

Density (calculated)

Absorption coefficient

$\mathrm{F}(000)$

Crystal color

Crystal size

Theta range for data collection

Index ranges

Reflections collected

Independent reflections

Completeness to theta $=24.71^{\circ}$

Absorption correction

Max. and min. transmission

Refinement method

Data / restraints / parameters

Goodness-of-fit on $\mathrm{F}^{2}$

Final R indices $[\mathrm{I}>2 \operatorname{sigma}(\mathrm{I})=1912$ data $]$

$\mathrm{R}$ indices (all data; $0.85 \AA$ )

Extinction coefficient

Largest diff. peak and hole kaw63 (Armando Ramirez)

$\mathrm{C}_{18} \mathrm{H}_{34} \mathrm{O}_{3}$

298.45

163(2) K

$0.71073 \AA$

Monoclinic

$P 2_{1} / c$

$\mathrm{a}=28.310(11) \AA \quad \alpha=90^{\circ}$.

$\mathrm{b}=6.229(2) \AA$

$\beta=100.580(7)^{\circ}$.

$\mathrm{c}=10.339(4) \AA$

1792.2(11) $\AA^{3}$

4

$1.106 \mathrm{Mg} / \mathrm{m}^{3}$

$0.073 \mathrm{~mm}^{-1}$

664

colorless

$0.27 \times 0.19 \times 0.13 \mathrm{~mm}^{3}$

1.46 to $24.71^{\circ}$.

$-33 \leq h \leq 33,-7 \leq k \leq 7,-12 \leq l \leq 12$

14089

$3031[\mathrm{R}(\mathrm{int})=0.0768]$

$99.2 \%$

Semi-empirical from equivalents

0.9906 and 0.9806

Full-matrix least-squares on $\mathrm{F}^{2}$

3031 / 9 / 337

1.049

$\mathrm{R} 1=0.0666, \mathrm{wR} 2=0.1615$

$\mathrm{R} 1=0.1155, \mathrm{wR} 2=0.1891$

$0.010(2)$

0.360 and -0.209 e. $\AA^{-3}$ 
Table 2. Atomic coordinates ( $\times 10^{4}$ ) and equivalent isotropic displacement parameters $\left(\AA^{2} \times 10^{3}\right)$ for 40. $U(e q)$ is defined as one third of the trace of the orthogonalized $U^{i j}$ tensor.

\begin{tabular}{lrrrr}
\hline & $\mathrm{x}$ & $\mathrm{y}$ & $\mathrm{z}$ & $\mathrm{U}(\mathrm{eq})$ \\
\hline $\mathrm{O}(1)$ & $2526(1)$ & $6780(4)$ & $5601(2)$ & $30(1)$ \\
$\mathrm{O}(2)$ & $2015(1)$ & $6345(4)$ & $5170(2)$ & $31(1)$ \\
$\mathrm{C}(1)$ & $2659(1)$ & $5354(5)$ & $6721(3)$ & $25(1)$ \\
$\mathrm{C}(2)$ & $3095(1)$ & $6401(5)$ & $7562(3)$ & $27(1)$ \\
$\mathrm{C}(3)$ & $3534(1)$ & $6388(5)$ & $6897(3)$ & $27(1)$ \\
$\mathrm{C}(4)$ & $3665(1)$ & $4101(5)$ & $6529(3)$ & $24(1)$ \\
$\mathrm{C}(5)$ & $3230(1)$ & $3144(6)$ & $5626(3)$ & $28(1)$ \\
$\mathrm{C}(6)$ & $2781(1)$ & $3145(5)$ & $6252(4)$ & $28(1)$ \\
$\mathrm{C}(7)$ & $2209(1)$ & $5307(6)$ & $7364(3)$ & $27(1)$ \\
$\mathrm{C}(8)$ & $1819(1)$ & $6448(5)$ & $6377(3)$ & $28(1)$ \\
$\mathrm{C}(9)$ & $1777(2)$ & $8809(6)$ & $6739(4)$ & $35(1)$ \\
$\mathrm{C}(10)$ & $1346(1)$ & $5249(5)$ & $5963(4)$ & $31(1)$ \\
$\mathrm{C}(11)$ & $4146(1)$ & $3944(5)$ & $6025(3)$ & $26(1)$ \\
$\mathrm{C}(12)$ & $4131(2)$ & $5247(6)$ & $4750(4)$ & $31(1)$ \\
$\mathrm{C}(13)$ & $4556(1)$ & $4748(7)$ & $7060(4)$ & $36(1)$ \\
$\mathrm{C}(14)$ & $4249(2)$ & $1580(6)$ & $5739(4)$ & $33(1)$ \\
$\mathrm{C}(15)$ & $965(1)$ & $5144(6)$ & $6863(4)$ & $37(1)$ \\
$\mathrm{C}(16)$ & $1189(2)$ & $4496(7)$ & $8258(5)$ & $45(1)$ \\
$\mathrm{C}(17)$ & $600(2)$ & $3420(7)$ & $6285(6)$ & $58(1)$ \\
$\mathrm{C}(18)$ & $693(2)$ & $7280(6)$ & $6881(5)$ & $50(1)$ \\
$\mathrm{O}(3)$ & $806(3)$ & $3939(12)$ & $8960(8)$ & $34(2)$ \\
$\mathrm{O}(4)$ & $375(3)$ & $3888(14)$ & $5276(7)$ & $27(2)$ \\
$\mathrm{O}(5)$ & $496(3)$ & $7903(11)$ & $5366(7)$ & $30(2)$ \\
- & & & \\
\hline
\end{tabular}


Table 3. Bond lengths $[\AA]$ and angles $\left[^{\circ}\right]$ for $\mathbf{4 0 .}$

\begin{tabular}{ll}
\hline $\mathrm{O}(1)-\mathrm{C}(1)$ & $1.452(4)$ \\
$\mathrm{O}(1)-\mathrm{O}(2)$ & $1.461(3)$ \\
$\mathrm{O}(2)-\mathrm{C}(8)$ & $1.456(4)$ \\
$\mathrm{C}(1)-\mathrm{C}(6)$ & $1.520(4)$ \\
$\mathrm{C}(1)-\mathrm{C}(2)$ & $1.522(5)$ \\
$\mathrm{C}(1)-\mathrm{C}(7)$ & $1.540(5)$ \\
$\mathrm{C}(2)-\mathrm{C}(3)$ & $1.524(5)$ \\
$\mathrm{C}(3)-\mathrm{C}(4)$ & $1.537(4)$ \\
$\mathrm{C}(4)-\mathrm{C}(5)$ & $1.522(5)$ \\
$\mathrm{C}(4)-\mathrm{C}(11)$ & $1.547(5)$ \\
$\mathrm{C}(5)-\mathrm{C}(6)$ & $1.530(5)$ \\
$\mathrm{C}(7)-\mathrm{C}(8)$ & $1.534(5)$ \\
$\mathrm{C}(8)-\mathrm{C}(10)$ & $1.525(5)$ \\
$\mathrm{C}(8)-\mathrm{C}(9)$ & $1.528(5)$ \\
$\mathrm{C}(10)-\mathrm{C}(15)$ & $1.548(5)$ \\
$\mathrm{C}(11)-\mathrm{C}(13)$ & $1.514(5)$ \\
$\mathrm{C}(11)-\mathrm{C}(14)$ & $1.541(5)$ \\
$\mathrm{C}(11)-\mathrm{C}(12)$ & $1.542(5)$ \\
$\mathrm{C}(15)-\mathrm{C}(16)$ & $1.519(6)$ \\
$\mathrm{C}(15)-\mathrm{C}(17)$ & $1.533(6)$ \\
$\mathrm{C}(15)-\mathrm{C}(18)$ & $1.539(5)$ \\
$\mathrm{C}(16)-\mathrm{O}(3)$ & $1.453(8)$ \\
$\mathrm{C}(17)-\mathrm{O}(4)$ & $1.155(8)$ \\
$\mathrm{C}(18)-\mathrm{O}(5)$ & $1.611(9)$ \\
$\mathrm{C}(1)-\mathrm{O}(1)-\mathrm{O}(2)$ & $103.1(2)$ \\
$\mathrm{C}(8)-\mathrm{O}(2)-\mathrm{O}(1)$ & $104.0(2)$ \\
$\mathrm{O}(1)-\mathrm{C}(1)-\mathrm{C}(6)$ & $109.9(3)$ \\
$\mathrm{O}(1)-\mathrm{C}(1)-\mathrm{C}(2)$ & $104.9(2)$ \\
$\mathrm{C}(6)-\mathrm{C}(1)-\mathrm{C}(2)$ & $111.2(3)$ \\
$\mathrm{O}(1)-\mathrm{C}(1)-\mathrm{C}(7)$ & $104.1(3)$ \\
$\mathrm{C}(6)-\mathrm{C}(1)-\mathrm{C}(7)$ & $112.0(3)$ \\
$\mathrm{C}(2)-\mathrm{C}(1)-\mathrm{C}(7)$ & $114.3(3)$ \\
$\mathrm{C}(1)-\mathrm{C}(2)-\mathrm{C}(3)$ & $112.6(3)$ \\
&
\end{tabular}




$\begin{array}{ll}\mathrm{C}(2)-\mathrm{C}(3)-\mathrm{C}(4) & 111.7(3) \\ \mathrm{C}(5)-\mathrm{C}(4)-\mathrm{C}(3) & 108.1(3) \\ \mathrm{C}(5)-\mathrm{C}(4)-\mathrm{C}(11) & 115.5(3) \\ \mathrm{C}(3)-\mathrm{C}(4)-\mathrm{C}(11) & 114.2(3) \\ \mathrm{C}(4)-\mathrm{C}(5)-\mathrm{C}(6) & 112.5(3) \\ \mathrm{C}(1)-\mathrm{C}(6)-\mathrm{C}(5) & 113.0(3) \\ \mathrm{C}(8)-\mathrm{C}(7)-\mathrm{C}(1) & 104.8(3) \\ \mathrm{O}(2)-\mathrm{C}(8)-\mathrm{C}(10) & 101.0(3) \\ \mathrm{O}(2)-\mathrm{C}(8)-\mathrm{C}(9) & 108.1(3) \\ \mathrm{C}(10)-\mathrm{C}(8)-\mathrm{C}(9) & 115.8(3) \\ \mathrm{O}(2)-\mathrm{C}(8)-\mathrm{C}(7) & 102.1(2) \\ \mathrm{C}(10)-\mathrm{C}(8)-\mathrm{C}(7) & 116.6(3) \\ \mathrm{C}(9)-\mathrm{C}(8)-\mathrm{C}(7) & 111.2(3) \\ \mathrm{C}(8)-\mathrm{C}(10)-\mathrm{C}(15) & 121.7(3) \\ \mathrm{C}(13)-\mathrm{C}(11)-\mathrm{C}(14) & 107.6(3) \\ \mathrm{C}(13)-\mathrm{C}(11)-\mathrm{C}(12) & 109.0(3) \\ \mathrm{C}(14)-\mathrm{C}(11)-\mathrm{C}(12) & 108.3(3) \\ \mathrm{C}(13)-\mathrm{C}(11)-\mathrm{C}(4) & 110.5(3) \\ \mathrm{C}(14)-\mathrm{C}(11)-\mathrm{C}(4) & 109.6(3) \\ \mathrm{C}(12)-\mathrm{C}(11)-\mathrm{C}(4) & 111.6(3) \\ \mathrm{C}(16)-\mathrm{C}(15)-\mathrm{C}(17) & 108.5(3) \\ \mathrm{C}(16)-\mathrm{C}(15)-\mathrm{C}(18) & 109.9(4) \\ \mathrm{C}(17)-\mathrm{C}(15)-\mathrm{C}(18) & 107.9(3) \\ \mathrm{C}(16)-\mathrm{C}(15)-\mathrm{C}(10) & 111.5(3) \\ \mathrm{C}(17)-\mathrm{C}(15)-\mathrm{C}(10) & 106.8(3) \\ \mathrm{C}(18)-\mathrm{C}(15)-\mathrm{C}(10) & 112.0(3) \\ \mathrm{O}(3)-\mathrm{C}(16)-\mathrm{C}(15) & 108.6(5) \\ \mathrm{O}(4)-\mathrm{C}(17)-\mathrm{C}(15) & 113.3(5) \\ \mathrm{C}(15)-\mathrm{C}(18)-\mathrm{O}(5) & 106.3(4) \\ & \end{array}$


Table 4. Anisotropic displacement parameters $\left(\AA^{2} \times 10^{3}\right)$ for 40. The anisotropic displacement factor exponent takes the form: $-2 \pi^{2}\left[h^{2} a^{* 2} U^{11}+\ldots+2 h k a^{*} b^{*} U^{12}\right]$

\begin{tabular}{|c|c|c|c|c|c|c|}
\hline & $\mathrm{U}^{11}$ & $\mathrm{U}^{22}$ & $U^{33}$ & $U^{23}$ & $\mathrm{U}^{13}$ & $\mathrm{U}^{12}$ \\
\hline $\mathrm{O}(1)$ & $32(1)$ & $33(1)$ & $27(1)$ & $13(1)$ & $8(1)$ & $1(1)$ \\
\hline $\mathrm{O}(2)$ & $33(1)$ & $37(1)$ & $24(1)$ & $5(1)$ & $7(1)$ & $2(1)$ \\
\hline $\mathrm{C}(1)$ & $37(2)$ & $20(2)$ & $17(2)$ & $4(1)$ & $5(2)$ & $0(1)$ \\
\hline $\mathrm{C}(2)$ & $37(2)$ & $20(2)$ & $26(2)$ & $-3(2)$ & $8(2)$ & $0(2)$ \\
\hline $\mathrm{C}(3)$ & $37(2)$ & $25(2)$ & $22(2)$ & $-7(2)$ & $7(2)$ & $-4(2)$ \\
\hline $\mathrm{C}(4)$ & $36(2)$ & $19(2)$ & $18(2)$ & $2(1)$ & $5(1)$ & $1(1)$ \\
\hline $\mathrm{C}(5)$ & $34(2)$ & $25(2)$ & $25(2)$ & $-7(2)$ & $7(2)$ & $-1(1)$ \\
\hline $\mathrm{C}(6)$ & $32(2)$ & $23(2)$ & $28(2)$ & $-4(2)$ & $6(2)$ & $-5(2)$ \\
\hline $\mathrm{C}(7)$ & $35(2)$ & $27(2)$ & $22(2)$ & $0(2)$ & $10(2)$ & $0(2)$ \\
\hline $\mathrm{C}(8)$ & $35(2)$ & $25(2)$ & $28(2)$ & $4(1)$ & $13(2)$ & $1(2)$ \\
\hline $\mathrm{C}(9)$ & $43(3)$ & $21(2)$ & $44(2)$ & $4(2)$ & $16(2)$ & $-2(2)$ \\
\hline $\mathrm{C}(10)$ & $37(2)$ & $22(2)$ & $34(2)$ & $7(2)$ & $9(2)$ & $1(2)$ \\
\hline $\mathrm{C}(11)$ & $37(2)$ & $20(2)$ & $20(2)$ & $1(1)$ & $6(2)$ & $2(1)$ \\
\hline $\mathrm{C}(12)$ & $42(2)$ & $27(2)$ & $29(2)$ & $6(2)$ & $14(2)$ & $1(2)$ \\
\hline $\mathrm{C}(13)$ & $32(2)$ & $40(2)$ & $37(2)$ & $-3(2)$ & $5(2)$ & $-4(2)$ \\
\hline $\mathrm{C}(14)$ & $45(2)$ & $25(2)$ & $32(2)$ & $1(2)$ & $13(2)$ & $6(2)$ \\
\hline $\mathrm{C}(15)$ & $33(2)$ & $25(2)$ & $56(3)$ & $15(2)$ & $16(2)$ & $4(2)$ \\
\hline$C(16)$ & $46(3)$ & $38(2)$ & $57(3)$ & $14(2)$ & $29(2)$ & $5(2)$ \\
\hline $\mathrm{C}(17)$ & $34(3)$ & $33(2)$ & 111(4) & $19(3)$ & $22(3)$ & $-2(2)$ \\
\hline $\mathrm{C}(18)$ & $38(3)$ & $34(2)$ & $83(4)$ & $18(2)$ & $25(2)$ & $8(2)$ \\
\hline $\mathrm{O}(3)$ & $49(5)$ & $26(4)$ & $35(4)$ & $4(3)$ & $25(4)$ & $0(3)$ \\
\hline $\mathrm{O}(4)$ & $21(4)$ & $25(4)$ & $31(4)$ & $-1(3)$ & $-5(3)$ & $7(3)$ \\
\hline $\mathrm{O}(5)$ & $27(4)$ & $28(4)$ & $33(4)$ & $4(3)$ & $0(4)$ & $1(3)$ \\
\hline
\end{tabular}


Table 5. Hydrogen coordinates ( $\left.\mathrm{x} 10^{4}\right)$ and isotropic displacement parameters $\left(\AA^{2} \times 10^{3}\right)$ for 40.

\begin{tabular}{|c|c|c|c|c|}
\hline & $\mathrm{x}$ & $\mathrm{y}$ & $\mathrm{z}$ & $\mathrm{U}(\mathrm{eq})$ \\
\hline $\mathrm{H}(3 \mathrm{C})$ & 732 & 5021 & 9366 & 52 \\
\hline $\mathrm{H}(4 \mathrm{~B})$ & 139 & 4635 & 5390 & 41 \\
\hline $\mathrm{H}(5 \mathrm{C})$ & 405 & 6787 & 4936 & 45 \\
\hline $\mathrm{H}(2 \mathrm{~A})$ & $3151(13)$ & $5510(60)$ & $8410(40)$ & $43(10)$ \\
\hline $\mathrm{H}(2 \mathrm{~B})$ & $2985(12)$ & $7910(60)$ & $7760(30)$ & $36(10)$ \\
\hline $\mathrm{H}(3 \mathrm{~A})$ & $3453(12)$ & $7240(60)$ & $6050(30)$ & 31(9) \\
\hline $\mathrm{H}(3 \mathrm{~B})$ & $3806(13)$ & $7090(60)$ & $7510(40)$ & $40(10)$ \\
\hline $\mathrm{H}(4 \mathrm{~A})$ & $3720(10)$ & $3240(50)$ & $7390(30)$ & $19(8)$ \\
\hline $\mathrm{H}(5 \mathrm{~A})$ & $3296(12)$ & $1620(60)$ & $5370(30)$ & $33(9)$ \\
\hline $\mathrm{H}(5 \mathrm{~B})$ & $3137(12)$ & $4040(60)$ & $4740(30)$ & $35(10)$ \\
\hline $\mathrm{H}(6 \mathrm{~A})$ & $2470(13)$ & $2570(60)$ & $5650(40)$ & $40(10)$ \\
\hline $\mathrm{H}(6 \mathrm{~B})$ & $2839(13)$ & $2150(60)$ & $7060(40)$ & $39(10)$ \\
\hline $\mathrm{H}(7 \mathrm{~A})$ & $2264(12)$ & $6030(60)$ & $8190(40)$ & $31(9)$ \\
\hline $\mathrm{H}(7 \mathrm{~B})$ & 2097(14) & $3770(70)$ & $7470(40)$ & $52(11)$ \\
\hline $\mathrm{H}(9 \mathrm{~A})$ & 1702(13) & $8990(60)$ & $7620(40)$ & $38(10)$ \\
\hline $\mathrm{H}(9 \mathrm{~B})$ & 2101(14) & $9400(60)$ & $6780(40)$ & $39(10)$ \\
\hline $\mathrm{H}(9 \mathrm{C})$ & 1541(15) & $9640(70)$ & $6080(40)$ & $54(12)$ \\
\hline $\mathrm{H}(10 \mathrm{~A})$ & $1449(11)$ & $3670(60)$ & $5830(30)$ & $30(9)$ \\
\hline $\mathrm{H}(10 \mathrm{~B})$ & 1190(11) & $6000(50)$ & $5070(30)$ & $21(8)$ \\
\hline $\mathrm{H}(12 \mathrm{~A})$ & $3823(15)$ & $4870(60)$ & $4030(40)$ & $51(12)$ \\
\hline $\mathrm{H}(12 \mathrm{~B})$ & 4442(13) & $5170(50)$ & $4480(30)$ & $33(10)$ \\
\hline $\mathrm{H}(12 \mathrm{C})$ & $4097(11)$ & $6820(60)$ & $4910(30)$ & $23(8)$ \\
\hline $\mathrm{H}(13 \mathrm{~A})$ & $4540(12)$ & $6240(70)$ & $7230(40)$ & $39(10)$ \\
\hline $\mathrm{H}(13 \mathrm{~B})$ & 4884(13) & $4590(50)$ & $6780(30)$ & 31(9) \\
\hline $\mathrm{H}(13 \mathrm{C})$ & $4594(14)$ & $3990(70)$ & $8000(40)$ & $58(12)$ \\
\hline $\mathrm{H}(14 \mathrm{~A})$ & $4608(13)$ & $1460(60)$ & $5560(30)$ & $36(10)$ \\
\hline $\mathrm{H}(14 \mathrm{~B})$ & $4228(13)$ & $660(60)$ & $6500(40)$ & $45(11)$ \\
\hline $\mathrm{H}(14 \mathrm{C})$ & 4032(13) & $920(60)$ & $4960(40)$ & $39(10)$ \\
\hline $\mathrm{H}(16 \mathrm{~A})$ & $940(20)$ & $4410(140)$ & $8760(70)$ & $54(5)$ \\
\hline $\mathrm{H}(16 \mathrm{~B})$ & 1339(13) & $3130(30)$ & $8260(40)$ & $54(5)$ \\
\hline
\end{tabular}




$\begin{array}{lclll}\mathrm{H}(16 \mathrm{C}) & 1424(11) & 5500(50) & 8650(40) & 54(5) \\ \mathrm{H}(17 \mathrm{~A}) & 540(30) & 3780(150) & 5380(20) & 54(5) \\ \mathrm{H}(17 \mathrm{~B}) & 325(9) & 3280(60) & 6690(40) & 54(5) \\ \mathrm{H}(17 \mathrm{C}) & 792(12) & 2160(40) & 6410(40) & 54(5) \\ \mathrm{H}(18 \mathrm{~A}) & 550(20) & 7990(110) & 6090(40) & 54(5) \\ \mathrm{H}(18 B) & 405(8) & 7070(60) & 7210(40) & 54(5) \\ \mathrm{H}(18 \mathrm{C}) & 895(12) & 8360(50) & 7340(40) & 54(5)\end{array}$

\section{Bibliography}

(1) Pangborn, A. B.; Giardello, M. A.; Grubbs, R. H.; Rosen, R. K.; Timmers, F. J. Organometallics. 1996, 15, 1518-1520.

(2) Patnaik, P. A Comprehensive Guide to the Hazardous Properties of Chemical Substances; Van Nostrand Reinhold: New York, 1992.

(3) Sharpless, K. B.; Verhoeven, T. R. Aldrichimica Acta. 1979, 12, 63.

(4) Begum, K.; Hamada, Y.; Kim, H.; Nagai, Y.; Ono, K.; Masuyama, A.; McCullough, K. J.; Nojima, M.; Tsuchiya, K.; Wataya, Y. J. Med. Chem. 2001, 44, 2357-2361.

(5) Corey, E. J.; Venkateswarlu, A. J. Am. Chem. Soc. 1972, 94, 6190-6191.

(6) Fujita, Y.; Shiono, M.; Susuki, S. Synthesis. 1983, 10, 804-806.

(7) Peng, Z.-H.; Woerpel, K. A. Org. Lett. 2000, 2, 1379-1381.

(8) Kantor, E. A.; Kiladze, T. K.; Mel'nitskii, I. A.; Mironov, I. V.; Rakhmankulov, D. L. Zhurnal Obshchei Khimii. 1981, 51, 2700-2704.

(9) Dussault, P. H.; Zope, U. Tetrahedron Lett. 1995, 36, 3655-3658.

(10) Carlsen, P. H. J.; Katsuki, T.; Martin, V.; Sharpless, K. B. J. Org. Chem. 1981, 46, 3936-3938.

(11) Hansen, D. E.; Moore, C. L.; Plourde, R.; Yuan, P.; Shoemaker, M. R. J. Org. Chem. 1995, 60, $5360-6364$. 


\section{Analytical data}

A. HPLC trace and ${ }^{1}$ H NMR spectra:
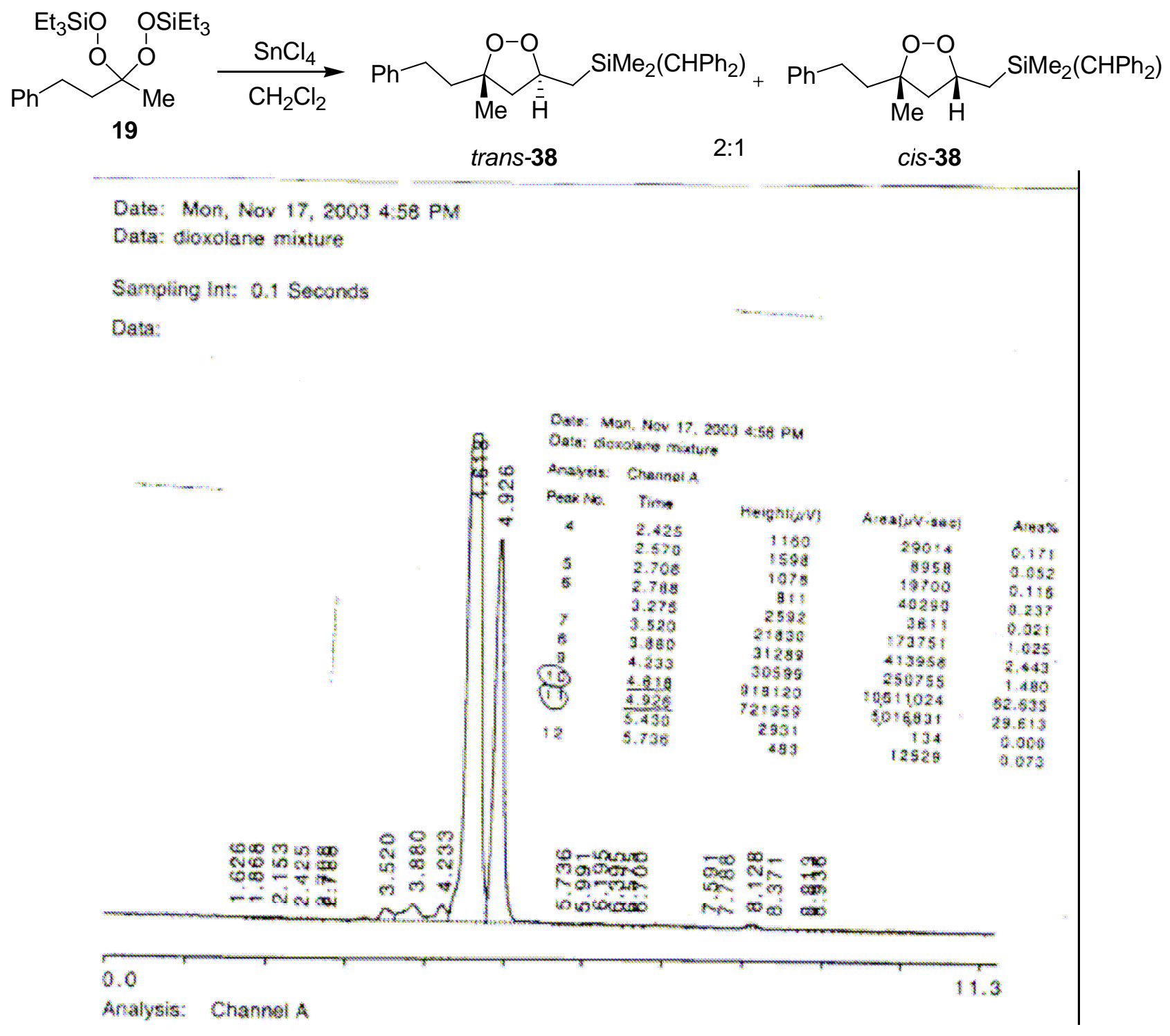


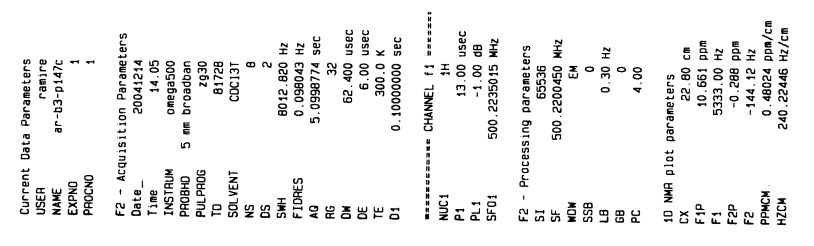

$68769^{\circ} \varepsilon$

¿E08G'

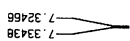

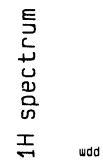

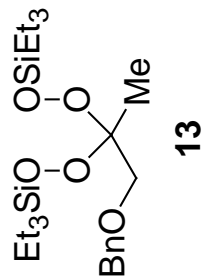

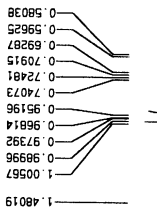



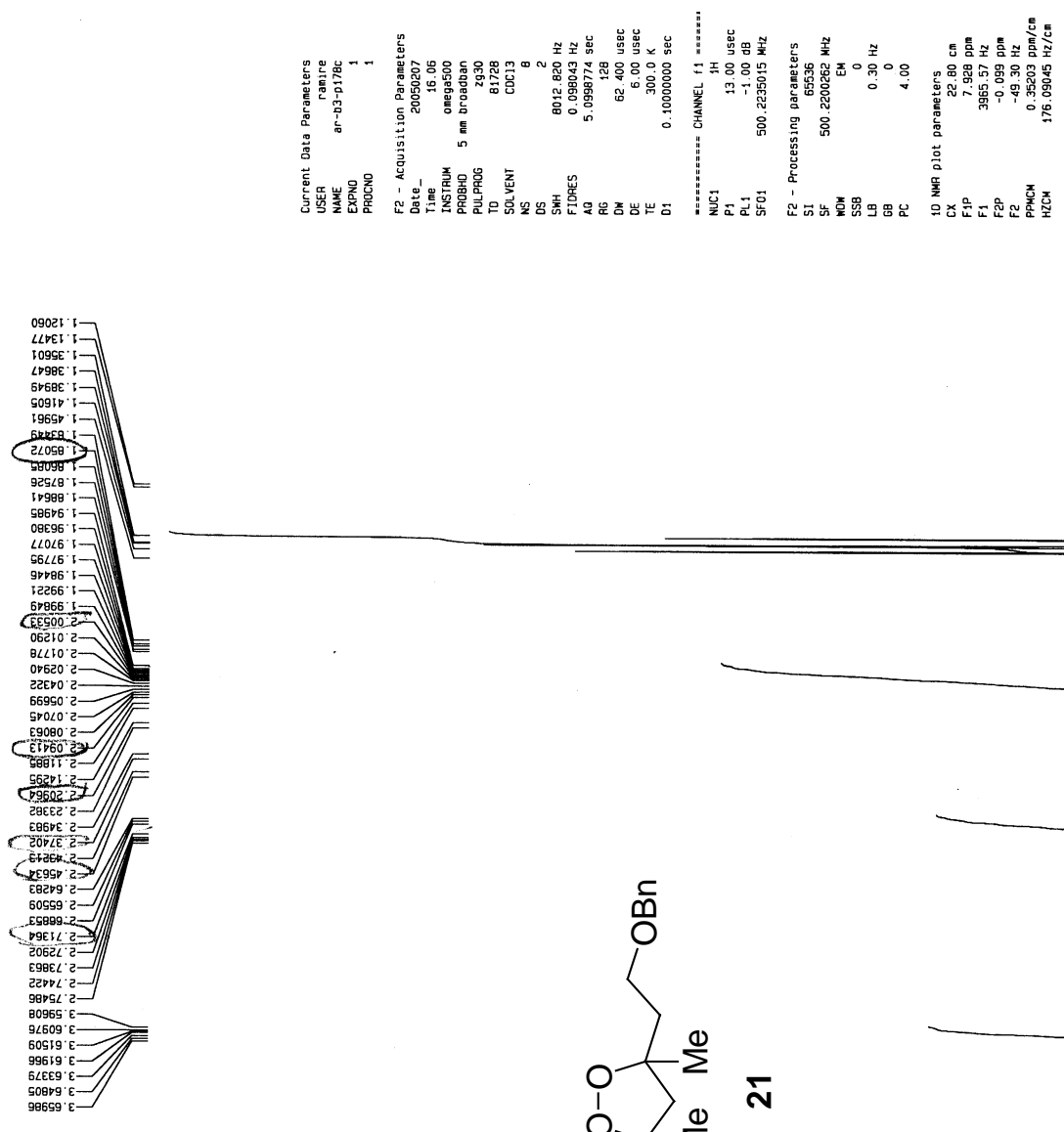

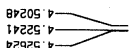
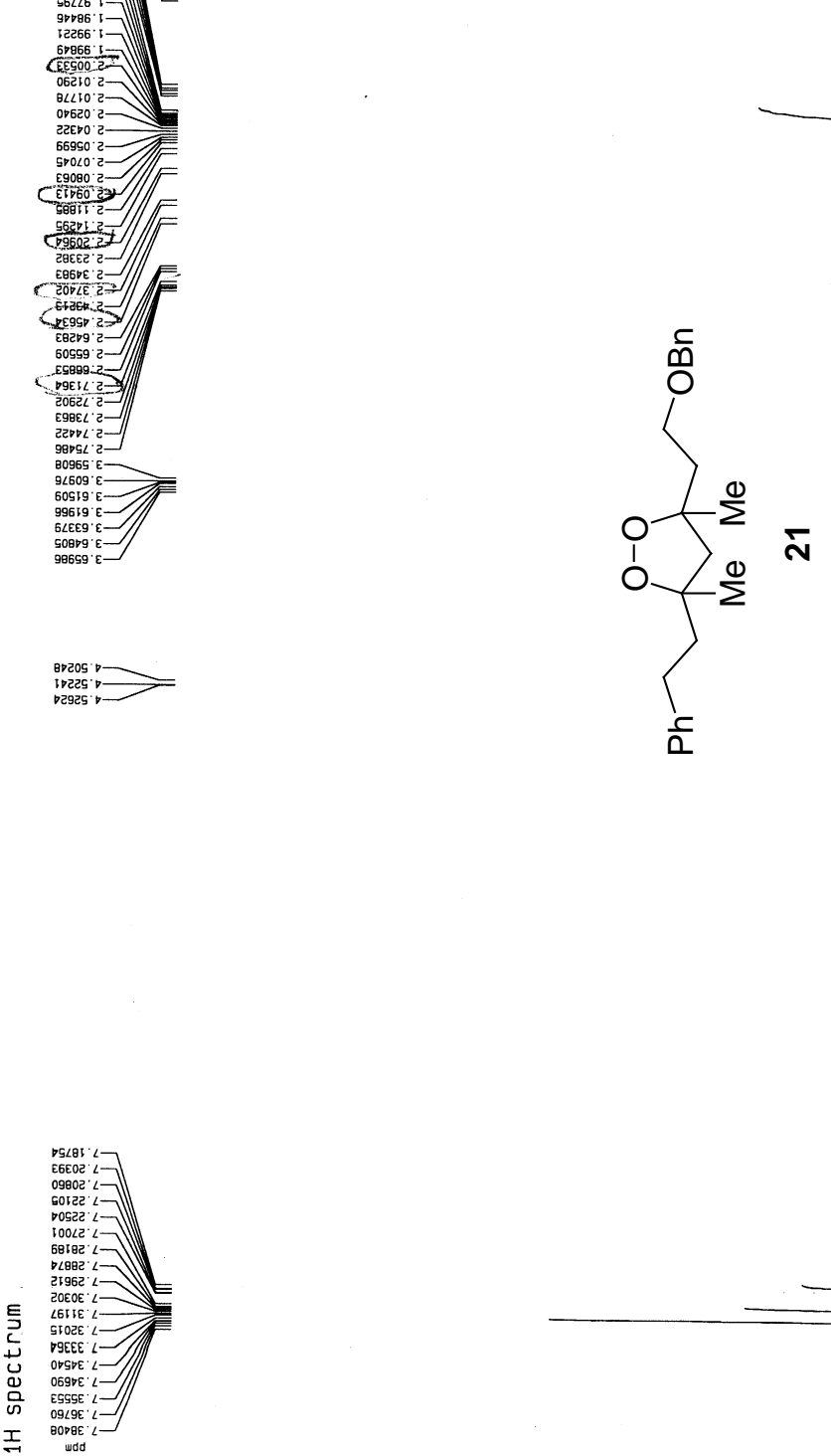

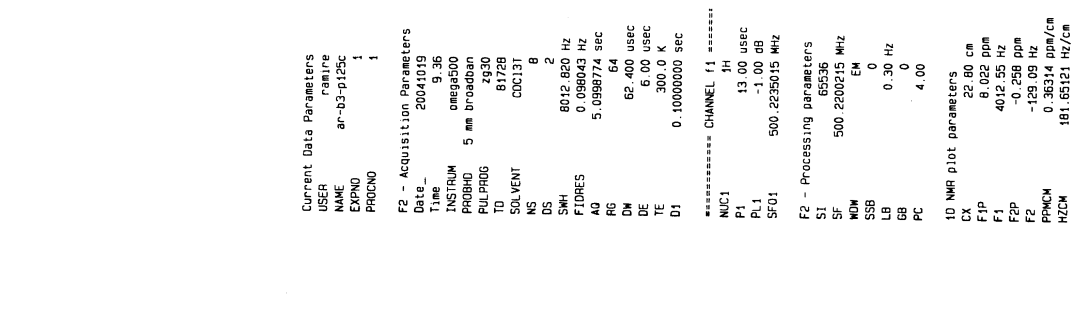

$$
\text { rroze: }
$$

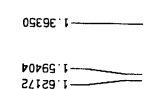

E9p66: $1-\square$
$69580^{2}-\square$

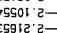

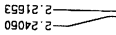

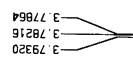
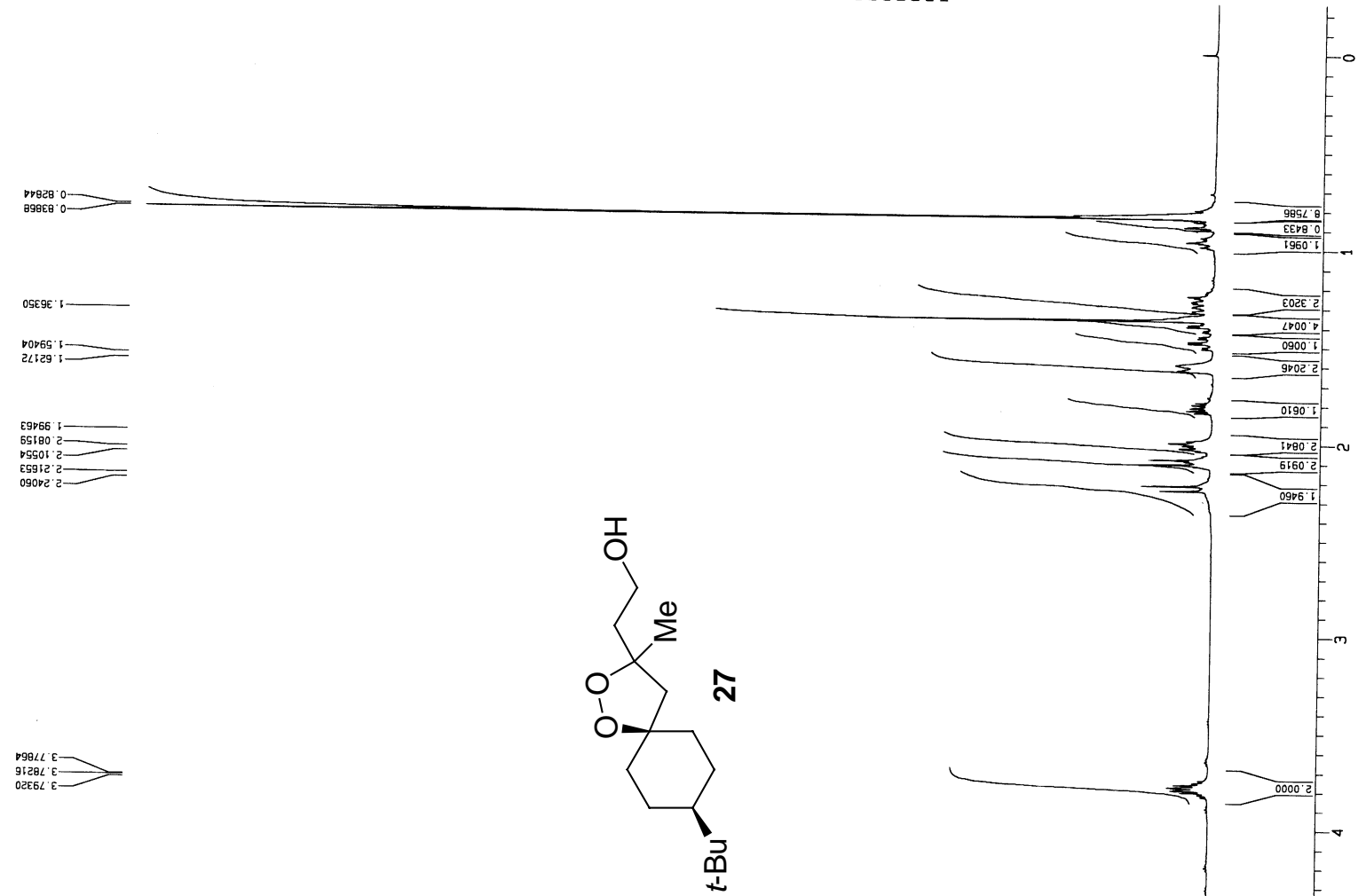

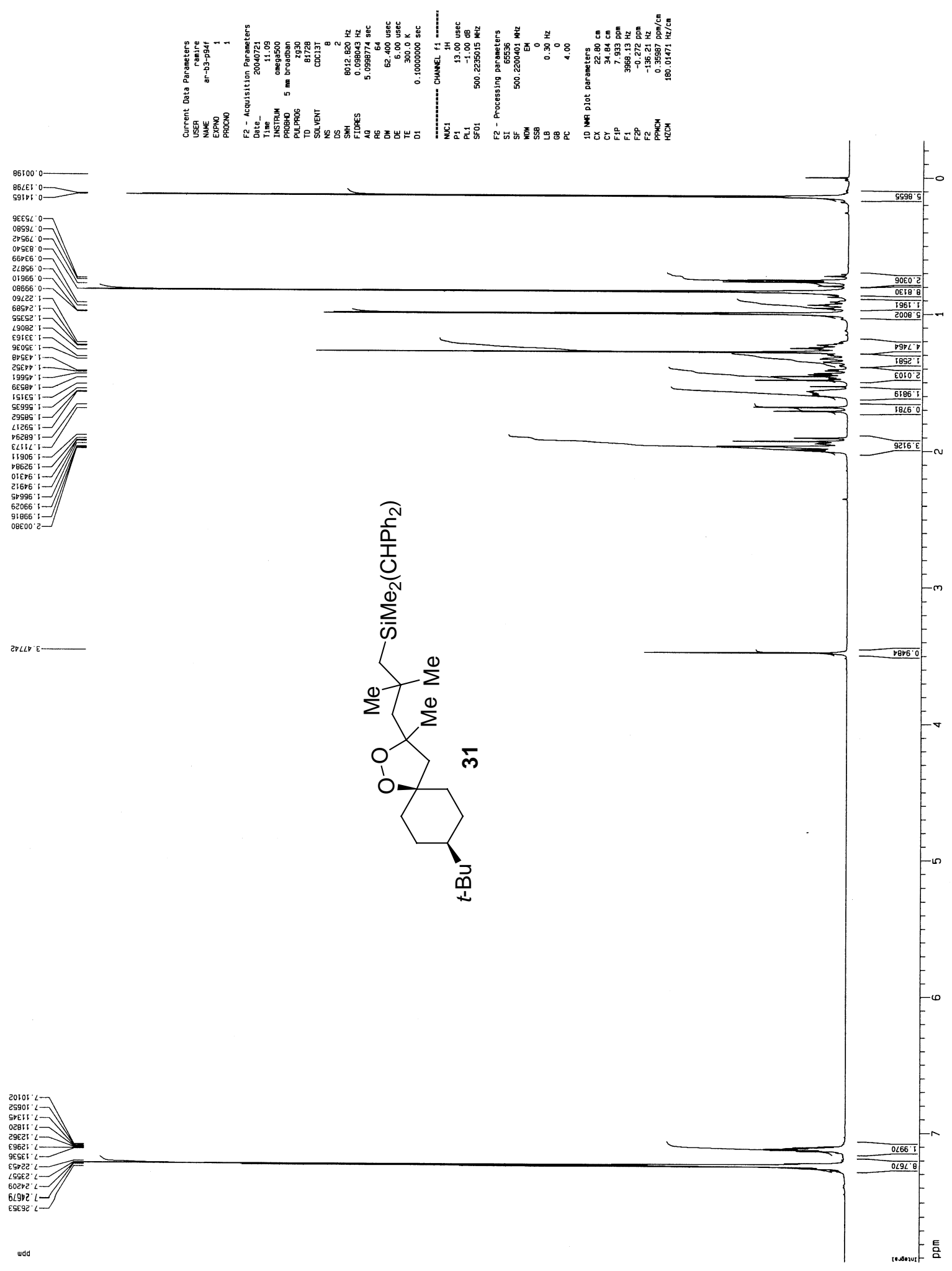

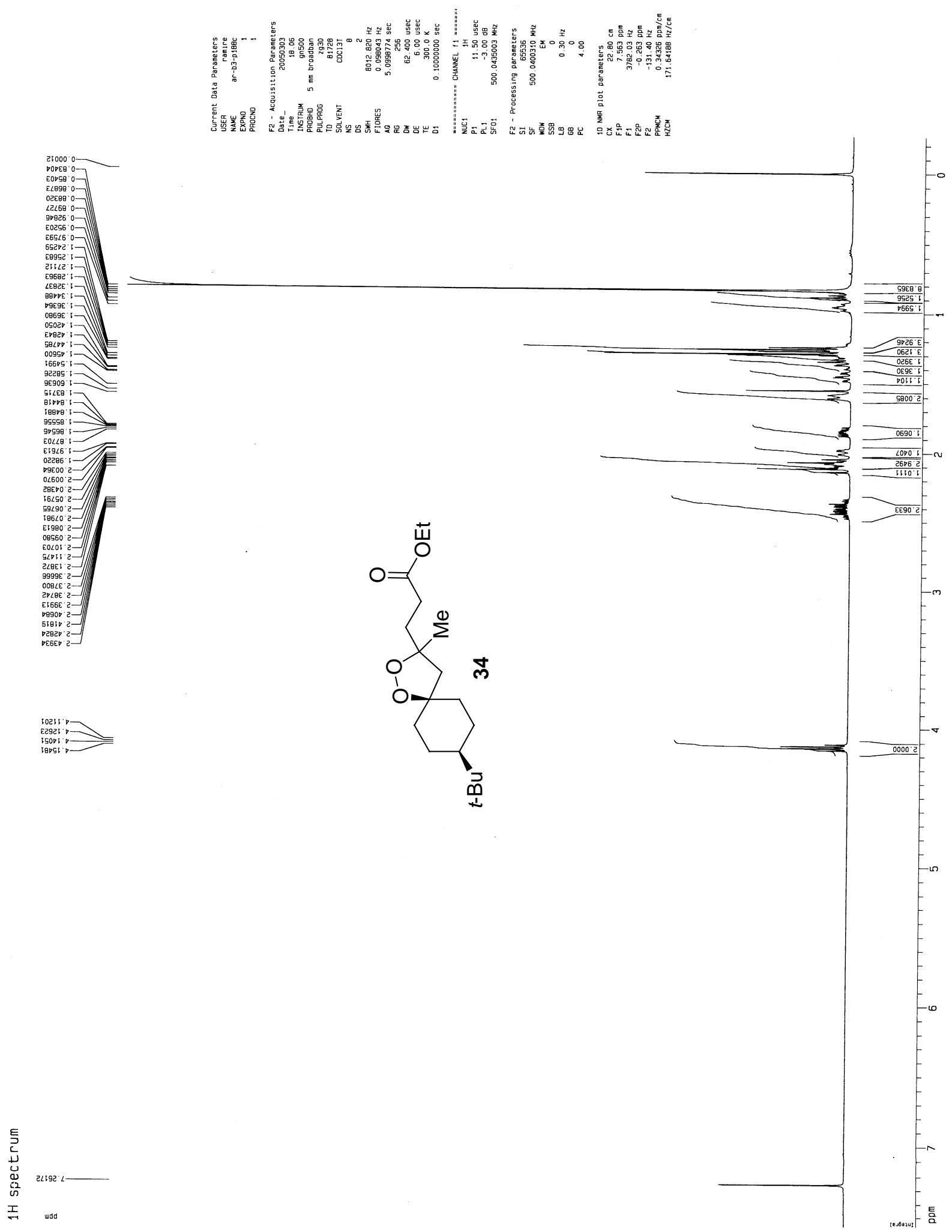

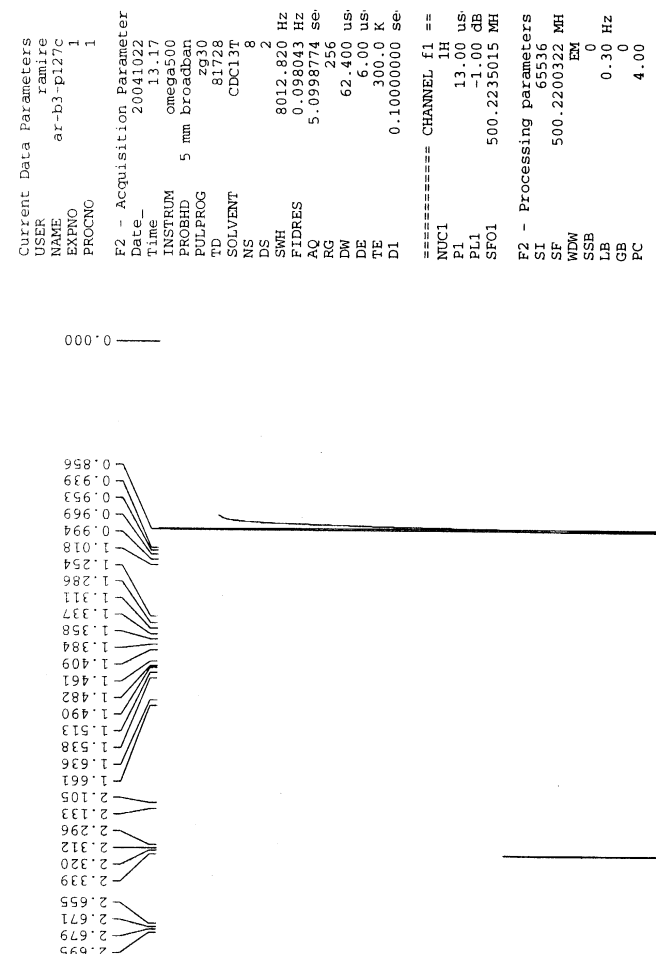

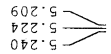
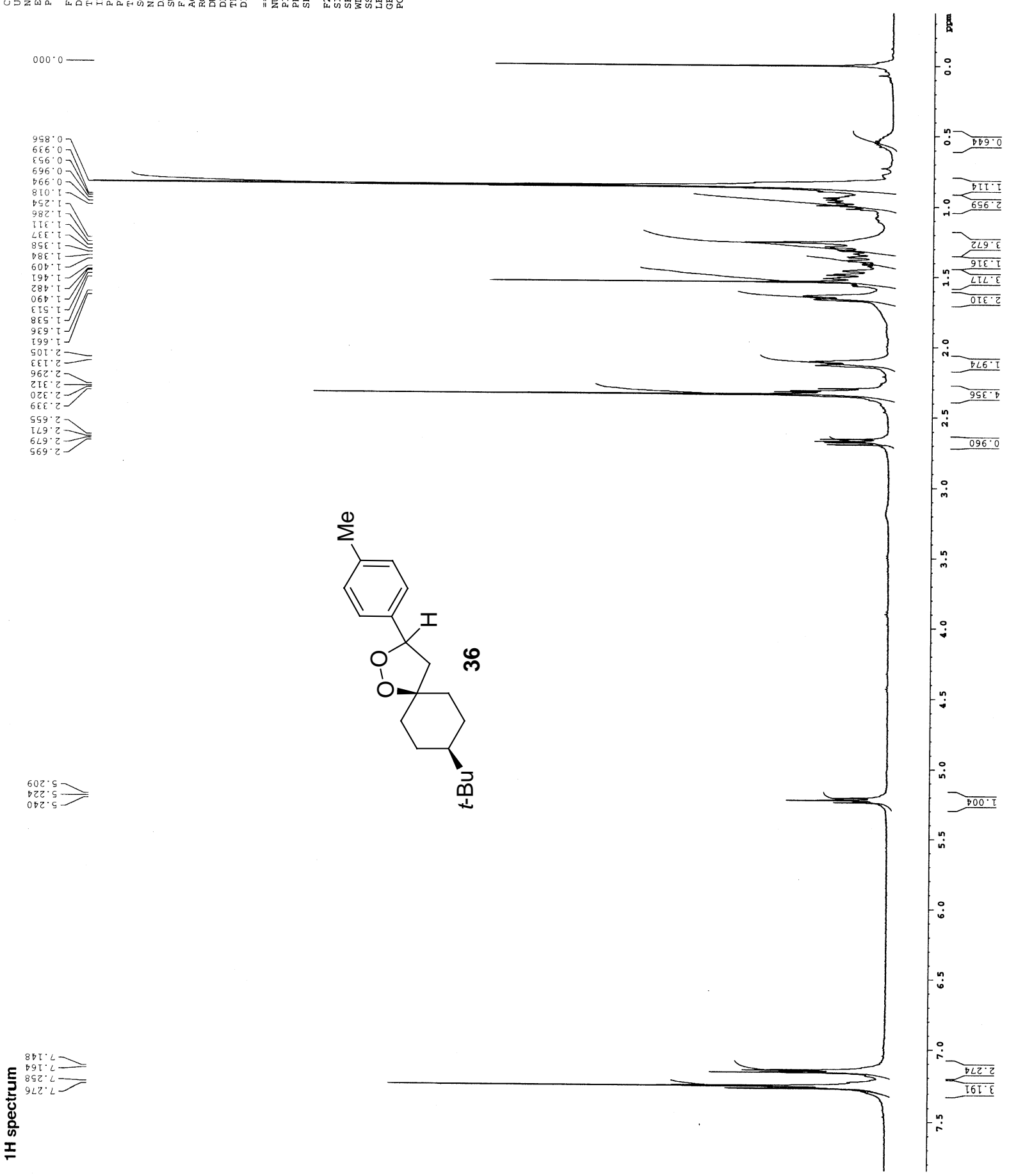


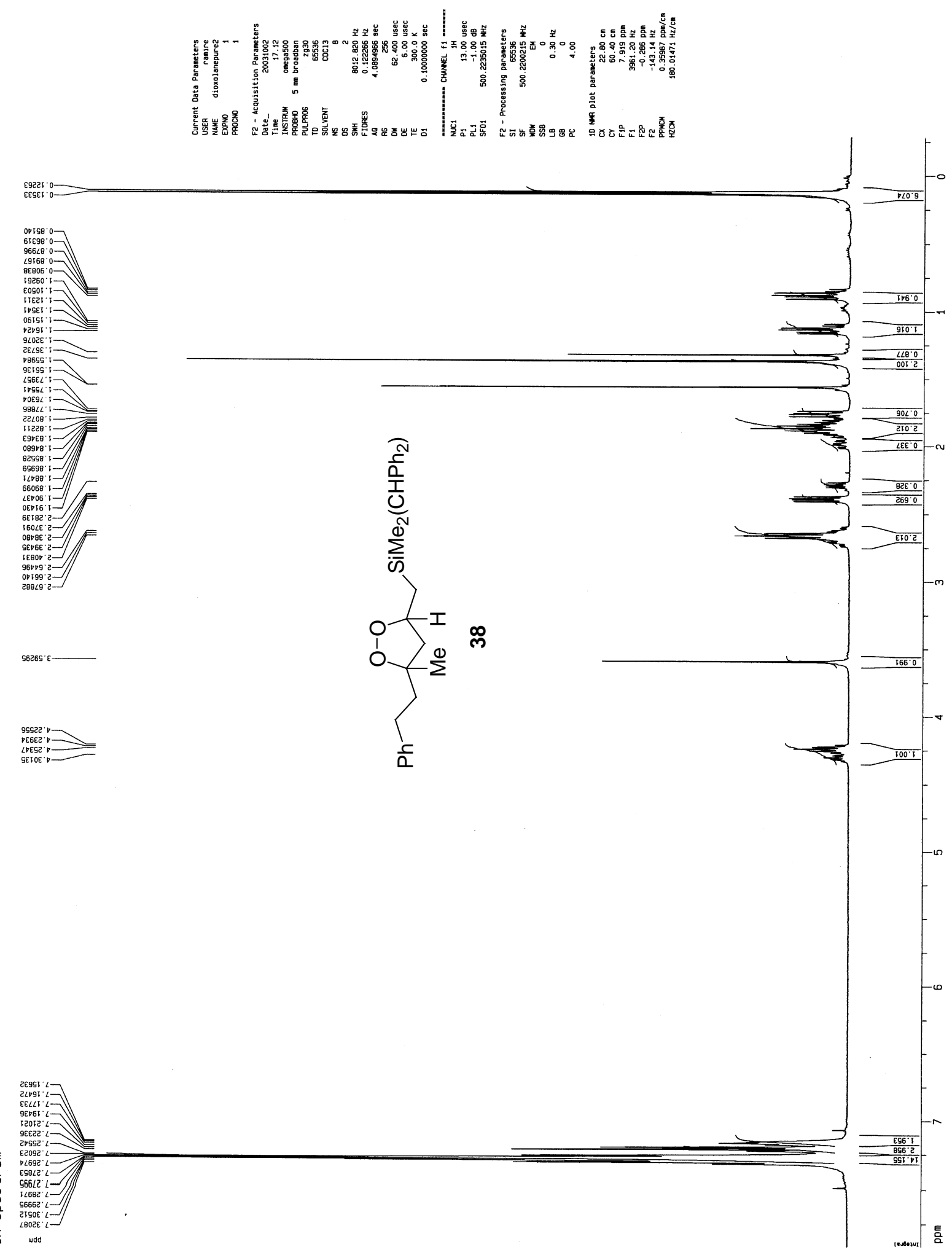

